(Version: 2019-01-25)

\title{
Sample paths of continuous-state branching processes with dependent immigration]
}

\author{
Zenghu Li \\ School of Mathematical Sciences, Beijing Normal University, \\ Beijing 100875, China.E-mail: lizh@bnu.edu.cn
}

\begin{abstract}
We prove the existence and pathwise uniqueness of the solution to a stochastic integral equation driven by Poisson random measures based on Kuznetsov measures for a continuous-state branching process. That gives a direct construction of the sample path of a continuous-state branching process with dependent immigration. The immigration rates depend on the population size via some functions satisfying a Yamada-Watanabe type condition. We only assume the existence of the first moment of the process. The existence of excursion law for the continuous-state branching process is not required. By special choices of the ingredients, we can make changes in the branching mechanism or construct models with competition.
\end{abstract}

Key words: continuous-state branching process; dependent immigration; stochastic equation; Poisson random measure; Yamada-Watanabe type condition.

MSC (2010) Subject Classification: 60J80, 60H10, 60H20

\section{Introduction}

The study of continuous-state branching processes (CB-processes) was started by Feller (1951), who noticed that a branching diffusion process may arise in a limit theorem of Galton-Watson discrete branching processes; see also Aliev and Shchurenkov (1982), Grimvall (1974) and Lamperti (1967a). A characterization of CB-processes by random time changes of Lévy processes was given by Lamperti (1967b). Continuous-state branching processes with immigration (CBI-processes) are natural generalizations of the CB-processes. The convergence of rescaled discrete branching processes with immigration to CBI-processes was studied in Aliev (1985), Kawazu and Watanabe (1971) and Li (2006, 2011). From a mathematical point of view, the continuous-state processes are usually easier to deal with because both their time and state spaces are smooth, and the distributions that appear are infinitely divisible. A continuous CBIprocess with subcritical branching mechanism was used by Cox et al. (1985) to describe the evolution of interest rates and it has been known in mathematical finance as the Cox-Ingersoll-Ross model (CIR-model). Compared with other financial models introduced before, the CIR-model

\footnotetext{
${ }^{1}$ Supported by the National Natural Science Foundation of China (No.11531001).
} 
is more appealing as it is positive (= nonnegative) and mean-reverting. For general treatments and backgrounds of CB- and CBI-processes, the reader may refer to Kyprianou (2014) and Li (2011, 2018b). More complicated continuous-state population models involving a competition mechanism were studied in Pardoux (2016), which extend the stochastic logistic growth model of Lambert (2005).

In this paper, we are interested in a class of Markov processes, which we call continuousstate branching processes with dependent immigration (CBDI-processes). By dependent immigration we mean the immigration rate depends on the state of the population via some function. This kind of immigration was studied in Dawson and Li (2003) and Fu and Li (2004) for measure-valued diffusions and extended in Li (2011) to general branching and immigration mechanisms. In those references, the immigration models were constructed in terms of stochastic equations driven by Poisson point measures on some path spaces. This approach is essential since the uniqueness of the corresponding martingale problems is usually unknown. In the references mentioned above, the immigration rate functions were assumed to be Lipschitz and the existence of some excursion laws of the corresponding measure-valued branching processes without immigration was required. In fact, some Poisson point measures based on the excursion laws were used there to represent the continuous part of the immigration.

The main purpose of this paper is to give a construction of the CBDI-process in terms of a stochastic equation of the type of $\mathrm{Li}$ (2011), but with non-Lipschitz immigration rate functions. A special case of the construction was given in the recent work of Li and Zhang (2019) by arguments of tightness and weak convergence, which require the existence of the second moment and the excursion law for the corresponding CB-process. We here replace the second moment assumption by the first moment one and remove the assumption on the existence of the excursion law. We focus on the one-dimensional model to simplify the presentations, but the arguments carry over to the measure-valued setting. In the stochastic equation considered here, the continuous immigration is represented by an increasing deterministic path and a Poisson point measure based on a Kuznetsov measure of the CB-process, which is slightly different from the equation in $\mathrm{Li}$ and Zhang (2019). The point of our approach is it gives a direct construction of the sample path of the CBDI-process with general branching and immigration mechanisms from those of the corresponding $\mathrm{CB}$-process without immigration. By special choices of the ingredients, we can make changes in the branching mechanism of the CB-process or construct a model with competition. These kinds of constructions have been proved useful for the study of some financial problems; see, e.g., Bernis and Scotti (2018+) and Jiao et al. (2017). A more precise description of our results is given as follows.

Suppose that $c \geq 0$ and $b$ are real constants and $m(\mathrm{~d} z)$ is a $\sigma$-finite measure on $(0, \infty)$ satisfying $\int_{0}^{\infty}\left(z \wedge z^{2}\right) m(\mathrm{~d} z)<\infty$. Let $\phi$ be a function on $[0, \infty)$ defined by

$$
\phi(\lambda)=b \lambda+c \lambda^{2}+\int_{(0, \infty)}\left(\mathrm{e}^{-z \lambda}-1+z \lambda\right) m(\mathrm{~d} z) .
$$

A Markov process with state space $[0, \infty)$ is called a $C B$-process with branching mechanism $\phi$ if it has transition semigroup $\left(Q_{t}\right)_{t \geq 0}$ given by

$$
\int_{[0, \infty)} \mathrm{e}^{-\lambda y} Q_{t}(x, \mathrm{~d} y)=\mathrm{e}^{-x v_{t}(\lambda)}, \quad \lambda \geq 0, x \geq 0,
$$


where $t \mapsto v_{t}(\lambda)$ is the unique positive solution of

$$
\frac{\partial}{\partial t} v_{t}(\lambda)=-\phi\left(v_{t}(\lambda)\right), \quad v_{0}(\lambda)=\lambda
$$

The family of functions $\left(v_{t}\right)_{t \geq 0}$ satisfies $v_{r+t}=v_{r} \circ v_{t}$ for $r, t \geq 0$ and is called the cumulant semigroup of the process. This semigroup has canonical representation

$$
v_{t}(\lambda)=h_{t} \lambda+\int_{(0, \infty)}\left(1-\mathrm{e}^{-\lambda z}\right) l_{t}(\mathrm{~d} z), \quad t \geq 0, \lambda \geq 0
$$

where $h_{t} \geq 0$ and $l_{t}(\mathrm{~d} z)$ is a $\sigma$-finite measure on $(0, \infty)$ satisfying $\int_{(0, \infty)} z l_{t}(\mathrm{~d} z)<\infty$; see Li (2011, 2018b).

A generalization of the CB-process is described as follows. Let $\beta \geq 0$ be a constant and $\nu(\mathrm{d} z)$ a $\sigma$-finite measure on $(0, \infty)$ satisfying $\int_{0}^{\infty}(1 \wedge z) \nu(\mathrm{d} z)<\infty$. Let $\psi$ be an immigration mechanism defined by

$$
\psi(\lambda)=\beta \lambda+\int_{(0, \infty)}\left(1-\mathrm{e}^{-\lambda z}\right) \nu(\mathrm{d} z), \quad \lambda \geq 0 .
$$

A Markov process with state space $[0, \infty)$ is called a CBI-process if it has transition semigroup $\left(P_{t}\right)_{t \geq 0}$ given by

$$
\int_{[0, \infty)} \mathrm{e}^{-\lambda y} P_{t}(x, \mathrm{~d} y)=\exp \left\{-x v_{t}(\lambda)-\int_{0}^{t} \psi\left(v_{s}(\lambda)\right) \mathrm{d} s\right\}
$$

where $\lambda \geq 0$ and $x \geq 0$.

From (1.2) and (1.6) we see that $\left(Q_{t}\right)_{t \geq 0}$ and $\left(P_{t}\right)_{t \geq 0}$ are Feller semigroups on $[0, \infty)$. Let $\mathscr{D}=C^{2}[0, \infty)$ be the set of bounded continuous real functions on $[0, \infty)$ with bounded continuous derivatives up to the second order. By Theorem 9.30 in Li (2011), the generator $L_{0}$ of the CB-process and the generator $L_{1}$ of the CBI-process are respectively defined by

$$
L_{0} f(x)=c x f^{\prime \prime}(x)-b x f^{\prime}(x)+x \int_{(0, \infty)}\left[f(x+z)-f(x)-z f^{\prime}(x)\right] m(\mathrm{~d} z),
$$

and

$$
L_{1} f(x)=L_{0} f(x)+\beta f^{\prime}(x)+\int_{(0, \infty)}[f(x+z)-f(x)] \nu(\mathrm{d} z), \quad x \geq 0, f \in \mathscr{D} .
$$

We are interested in a generalization of the generators defined by (1.7) and (1.8). Let $x \mapsto \beta(x)$ and $(x, z) \mapsto q(x, z)$ be positive Borel functions on $[0, \infty)$ and $[0, \infty) \times(0, \infty)$, respectively. We assume the following conditions:

(1.A) (linear growth condition) there is a constant $K \geq 0$ so that

$$
\beta(x)+\int_{(0, \infty)} q(x, z) z \nu(\mathrm{d} z) \leq K(1+x), \quad x \geq 0
$$


(1.B) (Yamada-Watanabe type condition) there is an increasing and concave function $u \mapsto r(u)$ on $[0, \infty)$ so that $\int_{0+} r(u)^{-1} \mathrm{~d} u=\infty$ and, for $x, y \geq 0$,

$$
|\beta(x)-\beta(y)|+\int_{(0, \infty)}|q(x, z)-q(y, z)| z \nu(\mathrm{d} z) \leq r(|x-y|) .
$$

By a $C B D I$-process we mean a Markov process in $[0, \infty)$ with generator $L$ defined by, for $x \geq 0$ and $f \in \mathscr{D}$,

$$
L f(x)=L_{0} f(x)+\beta(x) f^{\prime}(x)+\int_{(0, \infty)}[f(x+z)-f(x)] q(x, z) \nu(\mathrm{d} z) .
$$

Example 1.1 When $\beta(x) \equiv \beta$ and $q(x, z) \equiv 1$ are constants, the operator $(L, \mathscr{D})$ defined by (1.9) generates a classical CBI-process.

Example 1.2 If $\beta(x) \equiv \beta x$ and $q(x, z) \equiv x$ for some constant $\beta \geq 0$, then the operator $(L, \mathscr{D})$ defined by (1.9) generates a CB-process with branching mechanism

$$
\lambda \mapsto \phi(\lambda)-\beta \lambda-\int_{(0, \infty)}\left(1-\mathrm{e}^{-\lambda z}\right) \nu(\mathrm{d} z) .
$$

Then a change of the branching mechanism can be achieved by using dependent immigration.

Example 1.3 Let $x \mapsto G(x)$ be a positive function on $[0, \infty)$ satisfying the Yamada-Watanabe type condition and there is a constant $\beta>0$ so that $G(x) \leq \beta x$ for all $x \geq 0$. By setting $\beta(x) \equiv \beta x-G(x)$ and $q(x, z) \equiv 0$ in (1.9) we get, for $x \geq 0$ and $f \in \mathscr{D}$,

$$
L f(x)=L_{0} f(x)+\beta x f^{\prime}(x)-G(x) f^{\prime}(x) .
$$

Then $(L, \mathscr{D})$ generates a CB-process with competition; see, e.g., Berestycki et al. (2018), Lambert (2005) and Pardoux (2016). A more general class of population models, called continuousstate nonlinear branching processes, have been studied in Li (2018a) and Li et al. (2017+).

Let $\left(\Omega, \mathscr{F}, \mathscr{F}_{t}, \mathbf{P}\right)$ be a filtered probability space satisfying the usual conditions. Let $\{B(t)\}$ be a $\left(\mathscr{F}_{t}\right)$-Brownian motion and let $\{M(\mathrm{~d} s, \mathrm{~d} z, \mathrm{~d} u)\}$ and $\{N(\mathrm{~d} s, \mathrm{~d} z, \mathrm{~d} u)\}$ be $\left(\mathscr{F}_{t}\right)$-Poisson random measures on $(0, \infty)^{3}$ with intensities $\mathrm{d} s m(\mathrm{~d} z) \mathrm{d} u$ and $\mathrm{d} s \nu(\mathrm{d} z) \mathrm{d} u$, respectively. Suppose that $\{B(t)\},\{M(\mathrm{~d} s, \mathrm{~d} z, \mathrm{~d} u)\}$ and $\{N(\mathrm{~d} s, \mathrm{~d} z, \mathrm{~d} u)\}$ are independent of each other. Let $\{\tilde{M}(\mathrm{~d} s, \mathrm{~d} z, \mathrm{~d} u)\}$ be the compensated measure of $\{M(\mathrm{~d} s, \mathrm{~d} z, \mathrm{~d} u)\}$. By Theorem $5.1 \mathrm{in}$ Fu and Li (2010), for any $\mathscr{F}_{0}$-measurable positive random variable $y(0)$ there is a pathwise unique positive solution to the stochastic equation:

$$
\begin{aligned}
y(t)= & y(0)+\int_{0}^{t} \sqrt{2 c y(s-)} \mathrm{d} B(s)+\int_{0}^{t} \int_{0}^{\infty} \int_{0}^{y(s-)} z \tilde{M}(\mathrm{~d} s, \mathrm{~d} z, \mathrm{~d} u) \\
& +\int_{0}^{t}[\beta(y(s-))-b y(s-)] \mathrm{d} s+\int_{0}^{t} \int_{0}^{\infty} \int_{0}^{q(y(s-), z)} z N(\mathrm{~d} s, \mathrm{~d} z, \mathrm{~d} u) .
\end{aligned}
$$


Here and in the sequel, we understand that, for $b \geq a \geq 0$,

$$
\int_{a}^{b}=-\int_{b}^{a}=\int_{(a, b]} \text { and } \int_{a}^{\infty}=\int_{(a, \infty)} .
$$

The reader may refer to Ikeda and Watanabe (1989) and Situ (2005) for the theory of stochastic equations. We will show that a positive càdlàg process $\{y(t): t \geq 0\}$ is a weak solution of (1.10) if and only if it solves the martingale problem of $(L, \mathscr{D})$. Then (1.10) gives a construction of the CBDI-process. From this equation we see that the immigration of $\{y(t): t \geq 0\}$ involves two parts: the continuous part given by the drift $\beta(y(s-)) \mathrm{d} s$ and the discontinuous part determined by the intensity $q(y(s-), z)$ and Poisson random measure $N(\mathrm{~d} s, \mathrm{~d} z, \mathrm{~d} u)$. Stochastic equations in forms similar to (1.10) have also been studied in Bertoin and Le Gall (2006), Dawson and $\mathrm{Li}(2006,2012)$ and $\mathrm{Li}$ and Ma (2015).

Let $D[0, \infty)$ denote the space of positive càdlàg paths $w=\{w(t): t \geq 0\}$. For any $w \in D[0, \infty)$ let $\alpha(w)=\inf \{s \geq 0: w(s)>0\}$ and $\zeta(w)=\sup \{s \geq 0: w(s)>0\}$. Let $W$ be the set of paths $w \in D[0, \infty)$ such that $w(t)>0$ for $\alpha(w)<t<\zeta(w)$ and $w(t)=0$ for $t<\alpha(w)$ or $t \geq \zeta(w)$. Let $[0] \in W$ be the path that is constantly zero. On the space $W$ we define the $\sigma$-algebras $\mathscr{W}=\sigma(\{w(s): s \geq 0\})$ and $\mathscr{W}_{t}=\sigma(\{w(s): 0 \leq s \leq t\})$ for $t \geq 0$.

From (1.2) we see that zero is a trap for the CB-process. Let $\left(Q_{t}^{\circ}\right)_{t>0}$ be the restriction of the transition semigroup $\left(Q_{t}\right)_{t \geq 0}$ on $(0, \infty)$. For a $\sigma$-finite measure $\mu$ on $(0, \infty)$ write

$$
\mu Q_{t}^{\circ}(\mathrm{d} y)=\int_{(0, \infty)} \mu(\mathrm{d} x) Q_{t}^{\circ}(x, \mathrm{~d} y), \quad t \geq 0, y>0 .
$$

A family of $\sigma$-finite measures $\left(\kappa_{t}\right)_{t>0}$ on $(0, \infty)$ is called an entrance rule for $\left(Q_{t}^{\circ}\right)_{t \geq 0}$ if $\kappa_{r} Q_{t-r}^{\circ} \leq \kappa_{t}$ for all $t>r>0$ and $\kappa_{r} Q_{t-r}^{\circ} \rightarrow \kappa_{t}$ as $r \rightarrow t$.

Let $\left(l_{t}\right)_{t>0}$ be the family of $\sigma$-finite measures on $(0, \infty)$ determined by (1.4). By Theorems 3.13 and 3.15 in $\mathrm{Li}(2018 \mathrm{~b})$ we see that $\left(l_{t}\right)_{t>0}$ is an entrance rule for $\left(Q_{t}^{\circ}\right)_{t \geq 0}$, which is referred to as the canonical entrance rule of the CB-process. We shall give a simple and direct construction of the $\sigma$-finite measure $\mathbf{N}_{0}$ on $(W, \mathscr{W})$ such that, for $0<t_{1}<t_{2}<\cdots<t_{n}$ and $x_{1}, x_{2}, \ldots, x_{n} \in(0, \infty)$,

$$
\begin{aligned}
\mathbf{N}_{0}(\alpha(w) & \left.\leq t_{1}, w\left(t_{1}\right) \in \mathrm{d} x_{1}, w\left(t_{2}\right) \in \mathrm{d} x_{2}, \ldots, w\left(t_{n}\right) \in \mathrm{d} x_{n}, t_{n}<\zeta(w)\right) \\
& =l_{t_{1}}\left(\mathrm{~d} x_{1}\right) Q_{t_{2}-t_{1}}^{\circ}\left(x_{1}, \mathrm{~d} x_{2}\right) Q_{t_{3}-t_{2}}^{\circ}\left(x_{2}, \mathrm{~d} x_{3}\right) \cdots Q_{t_{n}-t_{n-1}}^{\circ}\left(x_{n-1}, \mathrm{~d} x_{n}\right) .
\end{aligned}
$$

We call $\mathrm{N}_{0}$ the canonical Kuznetsov measure of the CB-process. The existence of this measure is also a consequence of a general result on Markov processes; see Theorem 3.8 in Glover and Getoor (1987, p.63). The expression (1.12) intuitively means that the coordinate process $\{w(t): t>0\}$ under $\mathbf{N}_{0}$ is a Markov process in $(0, \infty)$ with transition semigroup $\left(Q_{t}^{\circ}\right)_{t \geq 0}$ and one-dimensional distributions $\left(l_{t}\right)_{t>0}$.

Let $\mathbf{Q}_{x}$ denote the distribution of the CB-process $\{x(t): t \geq 0\}$ with initial value $x(0)=$ $x \geq 0$. Let $\left\{\left(X_{t}, \mathscr{F}_{t}\right)\right\}$ be a CB-process with generator $L_{0}$ defined by (1.7) with $\mathbf{P}\left[X_{0}\right]<\infty$. Let $\left\{N_{0}(\mathrm{~d} s, \mathrm{~d} u, \mathrm{~d} w)\right\}$ be a Poisson random measure on $(0, \infty)^{2} \times W$ with intensity $\mathrm{d} s \mathrm{~d} u \mathbf{N}_{0}(\mathrm{~d} w)$ and $\left\{N_{1}(\mathrm{~d} s, \mathrm{~d} z, \mathrm{~d} u, \mathrm{~d} w)\right\}$ a Poisson random measure on $(0, \infty)^{3} \times W$ with intensity $\mathrm{d} s \nu(\mathrm{d} z)$ 
$\mathrm{d} u \mathbf{Q}_{z}(\mathrm{~d} w)$. Suppose that $\left\{\left(X_{t}, \mathscr{F}_{t}\right)\right\},\left\{N_{0}(\mathrm{~d} s, \mathrm{~d} u, \mathrm{~d} w)\right\}$ and $\left\{N_{1}(\mathrm{~d} s, \mathrm{~d} z, \mathrm{~d} u, \mathrm{~d} w)\right\}$ are defined on a complete probability space and are independent of each other. For $t \geq 0$ let $\mathscr{G}_{t}=\sigma\left(\mathscr{F}_{t} \cup \mathscr{H}_{t}\right)$, where

$$
\begin{aligned}
& \mathscr{H}_{t}=\sigma\left(\left\{N_{0}((0, s] \times B \times A), N_{1}((0, s] \times C \times A):\right.\right. \\
& \left.\left.0<s \leq t, B \in \mathscr{B}(0, \infty), C \in \mathscr{B}(0, \infty)^{2}, A \in \mathscr{W}_{t-s}\right\}\right) .
\end{aligned}
$$

We consider the stochastic integral equation

$$
\begin{aligned}
Y_{t}=X_{t} & +\int_{0}^{t} h_{t-s} \beta\left(Y_{s-}\right) \mathrm{d} s+\int_{0}^{t} \int_{0}^{\beta\left(Y_{s-}\right)} \int_{W} w(t-s) N_{0}(\mathrm{~d} s, \mathrm{~d} u, \mathrm{~d} w) \\
& +\int_{0}^{t} \int_{0}^{\infty} \int_{0}^{q\left(Y_{s-}, z\right)} \int_{W} w(t-s) N_{1}(\mathrm{~d} s, \mathrm{~d} z, \mathrm{~d} u, \mathrm{~d} w) .
\end{aligned}
$$

By a solution of (1.13) we mean a positive càdlàg process $\left\{Y_{t}: t \geq 0\right\}$ that is adapted to the filtration $\left(\mathscr{G}_{t}\right)$ and satisfies the equation a.s. for each $t \geq 0$.

The main result of this paper shows that there is a pathwise unique solution $\left\{Y_{t}: t \geq 0\right\}$ of (1.13) and $\left\{\left(Y_{t}, \mathscr{G}_{t}\right): t \geq 0\right\}$ is indeed a CBDI-process. Here the second and third terms on the right-hand side of the equation represent the continuous part of the immigration and the last term represents the discontinuous immigration. In Section 2, we provide the direct construction of the canonical Kuznetsov measure defined by (1.12). We also give a reformulation of the Markov property of the measure that is more convenient for our applications. In Section 3, we construct some inhomogeneous immigration processes with deterministic immigration rates. In Section 4, we construct immigration processes with predictable immigration rates and prove some useful properties of them. The existence and uniqueness of solution to the stochastic equation (1.13) are established in Section 5.

\section{The canonical Kuznetsov measure}

In this section, we give a simple and direct construction of the canonical Kuznetsov measure defined by (1.12). For notational convenience, we extend the definition of each path $w \in W$ by setting $w(s)=0$ for $s<0$. Recall that $\mathbf{Q}_{x}$ denotes the distribution on $(W, \mathscr{W})$ of the CBprocess $\{x(t): t \geq 0\}$ with $x(0)=x \geq 0$. For $r \geq 0$ and $w \in W$, we define $\rho_{r} w \in W$ by $\rho_{r} w(t)=w(t-r)$. Let $\mathbf{Q}_{x}^{(r)}$ denote the image of $\mathbf{Q}_{x}$ induced by the map $w \mapsto \rho_{r} w$. Then $\mathbf{Q}_{x}^{(r)}$ is supported by $\{w \in W: \alpha(w)=r, w(r)=x\}$. Given any $\sigma$-finite measure $\mu$ on $[0, \infty)$ let

$$
\mathbf{Q}_{\mu}^{(r)}(A)=\int_{[0, \infty)} \mathbf{Q}_{x}^{(r)}(A) \mu(\mathrm{d} x), \quad A \in \mathscr{W}
$$

In particular, if $\mu$ is a probability measure, then $\mathbf{Q}_{\mu}^{(0)}$ is the distribution on $(W, \mathscr{W})$ of the CBprocess with initial distribution $\mu$ and $\mathbf{Q}_{\mu}^{(r)}$ is the image of $\mathbf{Q}_{\mu}^{(0)}$ induced by the map $w \mapsto \rho_{r} w$.

Lemma 2.1 For any $s>r \geq 0$ and any positive $\mathscr{W}_{r}$-measurable function $F$ on $W$ we have $\mathbf{Q}_{\mu}^{(s)}(\{w \in W: F(w) \neq F([0])\})=0$. 
Proof. Let $0 \leq r_{1} \leq r_{2} \leq \cdots \leq r_{n} \leq r<s$ and let $f_{1}, f_{2}, \ldots, f_{n}$ be bounded Borel functions on $[0, \infty)$. For any $x \geq 0$ we have

$$
\begin{aligned}
\mathbf{Q}_{x}^{(s)} & {\left[f_{1}\left(w\left(r_{1}\right)\right) f_{2}\left(w\left(r_{2}\right)\right) \cdots f_{n}\left(w\left(r_{n}\right)\right)\right] } \\
& =\mathbf{Q}_{x}\left[f_{1}\left(w\left(r_{1}-s\right)\right) f_{2}\left(w\left(r_{2}-s\right)\right) \cdots f_{n}\left(w\left(r_{n}-s\right)\right)\right] \\
& =f_{1}(0) f_{2}(0) \cdots f_{n}(0) \\
& =f_{1}\left([0]\left(r_{1}\right)\right) f_{2}\left([0]\left(r_{2}\right)\right) \cdots f_{n}\left([0]\left(r_{n}\right)\right) .
\end{aligned}
$$

A monotone class argument shows $\mathbf{Q}_{x}^{(s)} F(w)=F([0])$ for any positive $\mathscr{W}_{r}$-measurable function $F$ on $W$, and so $\mathbf{Q}_{x}^{(s)} f(F(w))=f(F([0]))$ for any bounded Borel function $f$ on $[0, \infty)$. Then we have $\mathbf{Q}_{x}^{(s)}(\{w \in W: F(w) \neq F([0])\})=0$, which implies the desired result by (2.1).

Theorem 2.2 Let $\left(l_{t}\right)_{t>0}$ be the canonical entrance rule for the CB-process determined by (1.4). Then there is a unique $\sigma$-finite measure $\mathbf{N}_{0}$ on $(W, \mathscr{W})$ that does not charge the singleton $\{[0]\} \in$ $\mathscr{W}$ and satisfies (1.12). Moreover, we have:

(1) If $\phi^{\prime}(\infty)=\infty$, then $\mathbf{N}_{0}$ is supported by $\{w \in W: \alpha(w)=0, w(0)=0\}$.

(2) If $\delta:=\phi^{\prime}(\infty)<\infty$, then $\mathbf{N}_{0}$ is supported by $\{w \in W: \alpha(w)>0, w(\alpha(w))>0\}$ and has the representation

$$
\mathbf{N}_{0}(F)=\int_{0}^{\infty} \mathrm{e}^{-\delta s} \mathbf{Q}_{m}^{(s)}(F) \mathrm{d} s, \quad F \in \mathscr{W}
$$

Proof. In the case $\phi^{\prime}(\infty)=\infty$, we have $h_{t}=0$ for all $t>0$ and the result follows by Theorem $6.1 \mathrm{in} \mathrm{Li} \mathrm{(2018b).} \mathrm{In} \mathrm{the} \mathrm{case} \delta:=\phi^{\prime}(\infty)<\infty$, let us define the $\sigma$-finite measure $\mathbf{N}_{0}$ on $(W, \mathscr{W})$ by (2.2). Since $\mathbf{Q}_{m}^{(r)}$ is supported by $\{w \in W: \alpha(w)=r, w(r)>0\}$, we see that $\mathbf{N}_{0}$ is supported by $\{w \in W: \alpha(w)>0, w(\alpha(w))>0\}$. Let $f_{1}, \ldots, f_{n}$ be positive Borel functions on $[0, \infty)$ with $f_{1}(0)=\ldots=f_{n}(0)=0$. Then

$$
\begin{aligned}
\mathbf{N}_{0}\left[f_{1}\left(w\left(t_{1}\right)\right)\right] & =\mathbf{N}_{0}\left[\alpha(w) \leq t_{1}, f_{1}\left(w\left(t_{1}\right)\right)\right] \\
& =\int_{0}^{t_{1}} \mathrm{e}^{-\delta s} \mathbf{Q}_{m}^{(s)}\left[f_{1}\left(w\left(t_{1}\right)\right)\right] \mathrm{d} s \\
& =\int_{0}^{t_{1}} \mathrm{e}^{-\delta s} \mathbf{Q}_{m}^{(0)}\left[f_{1}\left(w\left(t_{1}-s\right)\right)\right] \mathrm{d} s \\
& =\int_{0}^{t_{1}} \mathrm{e}^{-\delta s} \mathrm{~d} s \int_{(0, \infty)} f_{1}(y) m Q_{t_{1}-s}^{\circ}(\mathrm{d} y) \\
& =\int_{(0, \infty)} f_{1}(y) l_{t_{1}}(\mathrm{~d} y),
\end{aligned}
$$

where the last equality follows by Theorem 3.15 in $\mathrm{Li}(2018 \mathrm{~b})$. Then 1.12 ) holds for $n=1$. From the Markov property of $\mathbf{Q}_{m}^{(s)}$ it follows that, for $n \geq 2$,

$$
\mathbf{N}_{0}\left[\alpha(w) \leq t_{1}, f_{1}\left(w\left(t_{1}\right)\right) \cdots f_{n-1}\left(w\left(t_{n-1}\right)\right) f_{n}\left(w\left(t_{n}\right)\right), t_{n}<\zeta(w)\right]
$$




$$
\begin{aligned}
& =\mathbf{N}_{0}\left[\alpha(w) \leq t_{1}, f_{1}\left(w\left(t_{1}\right)\right) \cdots f_{n-1}\left(w\left(t_{n-1}\right)\right) f_{n}\left(w\left(t_{n}\right)\right)\right] \\
& =\int_{0}^{t_{1}} \mathrm{e}^{-\delta s} \mathbf{Q}_{m}^{(s)}\left[f_{1}\left(w\left(t_{1}\right)\right) \cdots f_{n-1}\left(w\left(t_{n-1}\right)\right) f_{n}\left(w\left(t_{n}\right)\right)\right] \mathrm{d} s \\
& =\int_{0}^{t_{1}} \mathrm{e}^{-\delta s} \mathbf{Q}_{m}^{(0)}\left[f_{1}\left(w\left(t_{1}-s\right)\right) \cdots f_{n-1}\left(w\left(t_{n-1}-s\right)\right) f_{n}\left(w\left(t_{n}-s\right)\right)\right] \mathrm{d} s \\
& =\int_{0}^{t_{1}} \mathrm{e}^{-\delta s} \mathbf{Q}_{m}^{(0)}\left[f_{1}\left(w\left(t_{1}-s\right)\right) \cdots f_{n-1}\left(w\left(t_{n-1}-s\right)\right) Q_{t_{n}-t_{n-1}}^{\circ} f_{n}\left(w\left(t_{n-1}-s\right)\right)\right] \mathrm{d} s \\
& =\int_{0}^{t_{1}} \mathrm{e}^{-\delta s} \mathbf{Q}_{m}^{(s)}\left[f_{1}\left(w\left(t_{1}\right)\right) \cdots f_{n-1}\left(w\left(t_{n-1}\right)\right) Q_{t_{n}-t_{n-1}}^{\circ} f_{n}\left(w\left(t_{n-1}\right)\right)\right] \mathrm{d} s \\
& =\mathbf{N}_{0}\left[f_{1}\left(w\left(t_{1}\right)\right) \cdots f_{n-1}\left(w\left(t_{n-1}\right)\right) Q_{t_{n}-t_{n-1}}^{\circ} f_{n}\left(w\left(t_{n-1}\right)\right)\right] .
\end{aligned}
$$

Then we get (1.12) by induction, which determines the measure $\mathrm{N}_{0}$ uniquely by the measure extension theorem.

Theorem 2.3 Let $t \geq r>0$ and let $F$ be a positive $\mathscr{W}_{r}$-measurable function on $W$. Then for any $\lambda \geq 0$ we have

$$
\mathbf{N}_{0}\left[F(w)\left(1-\mathrm{e}^{-\lambda w(t)}\right)\right]=\mathbf{N}_{0}\left[F(w)\left(1-\mathrm{e}^{-v_{t-r}(\lambda) w(r)}\right)\right]+F([0])\left[h_{r} v_{t-r}(\lambda)-h_{t} \lambda\right] .
$$

Proof. In the case $\phi^{\prime}(\infty)=\infty$, we have $h_{r}=h_{t}=0$ and (2.3) follows from (1.12). In the case $\delta:=\phi^{\prime}(\infty)<\infty$, the measure $\mathbf{N}_{0}$ is given by (2.2). By Lemma 2.1. for any $s>r$ we have $\mathbf{Q}_{m}^{(s)}(\{w \in W: F(w) \neq F([0])\})=0$. Then we use the Markov property of $\mathbf{Q}_{m}^{(s)}$ to see

$$
\begin{aligned}
\mathbf{N}_{0}\left[F(w)\left(1-\mathrm{e}^{-\lambda w(t)}\right)\right] & =\mathbf{N}_{0}\left[\alpha(w) \leq t, F(w)\left(1-\mathrm{e}^{-\lambda w(t)}\right)\right] \\
& =\int_{0}^{t} \mathrm{e}^{-\delta s} \mathbf{Q}_{m}^{(s)}\left[F(w)\left(1-\mathrm{e}^{-\lambda w(t)}\right)\right] \mathrm{d} s \\
& =\int_{0}^{r} \mathrm{e}^{-\delta s} \mathbf{Q}_{m}^{(s)}\left[F(w)\left(1-\mathrm{e}^{-v_{t-r}(\lambda) w(r)}\right)\right] \mathrm{d} s \\
& \quad+\int_{r}^{t} \mathrm{e}^{-\delta s} \mathbf{Q}_{m}^{(s)}\left[F([0])\left(1-\mathrm{e}^{-\lambda w(t)}\right)\right] \mathrm{d} s \\
= & \mathbf{N}_{0}\left[F(w)\left(1-\mathrm{e}^{-v_{t-s}(\lambda) w(r)}\right)\right] \\
& \quad+F([0]) \int_{r}^{t} \mathrm{e}^{-\delta s} \mathrm{~d} s \int_{(0, \infty)}\left(1-\mathrm{e}^{-x v_{t-s}(\lambda)}\right) m(\mathrm{~d} x) \\
& =\mathbf{N}_{0}\left[F(w)\left(1-\mathrm{e}^{-v_{t-s}(\lambda) w(r)}\right)\right]+F([0]) \mathrm{e}^{-\delta r}\left[v_{t-r}(\lambda)-\mathrm{e}^{-\delta(t-r)} \lambda\right],
\end{aligned}
$$

where the last equality holds by Theorem 3.15 of Li (2018b).

The existence of Markovian measures determined by entrance rules was first noticed by Kuznetsov (1974). In the setting of Borel right Markov processes, it was proved in Glover and Getoor (1987). The relation (2.3) gives a reformulation of the Markov property of the canonical Kuznetsov measure, which is more convenient than (1.12) for the application in the next section. In the special case of $\phi^{\prime}(\infty)=\infty$, we have $h_{t}=0$ for every $t>0$ and the canonical Kuznetsov measure $\mathbf{N}_{0}$ is known as the excursion law of the CB-process. In that special case, the property (2.3) is already known; see, e.g., the proof of Theorem 8.24 in Li (2011). 


\section{Deterministic immigration rates}

In this section, we give constructions of some inhomogeneous CBI-processes with deterministic immigration rates from random paths selected by Poisson point measures. The reader may refer to $\operatorname{Li}(2011,2018 \mathrm{~b})$ for similar constructions. Let $\left\{\left(X_{t}, \mathscr{F}_{t}\right)\right\},\left\{N_{0}(\mathrm{~d} s, \mathrm{~d} u, \mathrm{~d} w)\right\}$ and $\left\{N_{1}(\mathrm{~d} s, \mathrm{~d} z, \mathrm{~d} u, \mathrm{~d} w)\right\}$ be as in the introduction.

Theorem 3.1 Let $s \mapsto \rho(s)$ be a positive locally integrable function on $[0, \infty)$. For $t \geq 0$ let

$$
Y_{t}=X_{t}+\int_{0}^{t} h_{t-s} \rho(s) \mathrm{d} s+\int_{0}^{t} \int_{0}^{\rho(s)} \int_{W} w(t-s) N_{0}(\mathrm{~d} s, \mathrm{~d} u, \mathrm{~d} w)
$$

and let $\mathscr{G}_{t}=\sigma\left(\mathscr{F}_{t} \cup \mathscr{H}_{t}^{0}\right)$, where

$$
\mathscr{H}_{t}^{0}=\sigma\left(\left\{N_{0}((0, s] \times B \times A): 0<s \leq t, B \in \mathscr{B}(0, \infty), A \in \mathscr{W}_{t-s}\right\}\right) .
$$

Then $\left\{\left(Y_{t}, \mathscr{G}_{t}\right): t \geq 0\right\}$ is a Markov process in $[0, \infty)$ with inhomogeneous transition semigroup $\left(P_{r, t}^{\rho}\right)_{t \geq r \geq 0}$ given by

$$
\int_{[0, \infty)} \mathrm{e}^{-\lambda y} P_{r, t}^{\rho}(x, \mathrm{~d} y)=\exp \left\{-x v_{t-r}(\lambda)-\int_{r}^{t} v_{t-s}(\lambda) \rho(s) \mathrm{d} s\right\} .
$$

Proof. Let $Z_{t}=Y_{t}-X_{t}$ for $t \geq 0$. We first prove that $\left\{\left(Z_{t}, \mathscr{H}_{t}^{0}\right)\right\}$ is a Markov process with transition semigroup $\left(P_{r, t}^{\rho}\right)_{t \geq r \geq 0}$. Let $t \geq r \geq \tau \geq 0$ and let $F$ be a positive function on $(0, \infty)^{2} \times W$ measurable with respect to $\mathscr{B}(0, \tau] \times \mathscr{B}(0, \infty) \times \mathscr{W}_{r-\tau}$. It suffices to show, for any $\lambda \geq 0$,

$$
\begin{array}{r}
\mathbf{P}\left[\exp \left\{-\int_{0}^{\tau} \int_{0}^{\infty} \int_{W} F(s, u, w) N_{0}(\mathrm{~d} s, \mathrm{~d} u, \mathrm{~d} w)-\lambda Z_{t}\right\}\right] \\
=\mathbf{P}\left[\operatorname { e x p } \left\{-\int_{0}^{\tau} \int_{0}^{\infty} \int_{W} F(s, u, w) N_{0}(\mathrm{~d} s, \mathrm{~d} u, \mathrm{~d} w)\right.\right. \\
\left.\left.\quad-v_{t-r}(\lambda) Z_{r}-\int_{r}^{t} v_{t-s}(\lambda) \rho(s) \mathrm{d} s\right\}\right] .
\end{array}
$$

Since $\left\{1_{\{w=[0]\}} N_{0}(\mathrm{~d} s, \mathrm{~d} u, \mathrm{~d} w)\right\}$ is independent of $\left\{1_{\{w \neq[0]\}} N_{0}(\mathrm{~d} s, \mathrm{~d} u, \mathrm{~d} w)\right\}$ and $\left\{Z_{t}\right\}$, we can assume $F(s, u,[0])=0$ in the following calculations. Writing $G(s, u, w)=F(s, u, w) 1_{\{s \leq \tau\}}+$ $\lambda w(t-s) 1_{\{u \leq \rho(s)\}}$, we have

$$
\begin{aligned}
\mathbf{P}\left[\exp \left\{-\int_{0}^{\tau} \int_{0}^{\infty} \int_{W} F(s, u, w) N_{0}(\mathrm{~d} s, \mathrm{~d} u, \mathrm{~d} w)-\lambda Z_{t}\right\}\right] \\
=\mathbf{P}\left[\exp \left\{-\lambda \int_{0}^{t} h_{t-s} \rho(s) \mathrm{d} s-\int_{0}^{t} \int_{0}^{\infty} \int_{W} G(s, u, w) N_{0}(\mathrm{~d} s, \mathrm{~d} u, \mathrm{~d} w)\right\}\right] \\
=\exp \left\{-\lambda \int_{0}^{t} h_{t-s} \rho(s) \mathrm{d} s-\int_{0}^{t} \mathrm{~d} s \int_{0}^{\infty} \mathbf{N}_{0}\left(1-\mathrm{e}^{-G(s, u, w)}\right) \mathrm{d} u\right\} \\
=\exp \left\{-\lambda \int_{0}^{t} h_{t-s} \rho(s) \mathrm{d} s-\int_{0}^{t} \mathrm{~d} s \int_{0}^{\infty} \mathbf{N}_{0}\left(1-\mathrm{e}^{-F(s, u, w) 1_{\{s \leq \tau\}}}\right) \mathrm{d} u\right\}
\end{aligned}
$$




$$
\begin{aligned}
& \cdot \exp \left\{-\int_{0}^{t} \mathrm{~d} s \int_{0}^{\infty} \mathrm{N}_{0}\left[\mathrm{e}^{-F(s, u, w) 1_{\{s \leq \tau\}}}\left(1-\mathrm{e}^{\left.-\lambda w(t-s) 1_{\{u \leq \rho(s)\}}\right)}\right] \mathrm{d} u\right\}\right. \\
= & \exp \left\{-\lambda \int_{0}^{t} h_{t-s} \rho(s) \mathrm{d} s-\int_{0}^{t} \mathrm{~d} s \int_{0}^{\infty} \mathrm{N}_{0}\left(1-\mathrm{e}^{-F(s, u, w) 1_{\{s \leq \tau\}}}\right) \mathrm{d} u\right\} \\
\cdot & \exp \left\{-\int_{0}^{r} \mathrm{~d} s \int_{0}^{\rho(s)} \mathrm{N}_{0}\left[\mathrm{e}^{-F(s, u, w) 1_{\{s \leq \tau\}}}\left(1-\mathrm{e}^{-\lambda w(t-s)}\right)\right] \mathrm{d} u\right\} \\
\cdot & \exp \left\{-\int_{r}^{t} \mathrm{~d} s \int_{0}^{\rho(s)} \mathbf{N}_{0}\left(1-\mathrm{e}^{-\lambda w(t-s)}\right) \mathrm{d} u\right\},
\end{aligned}
$$

where, by Theorem 2.3 ,

$$
\begin{aligned}
& \mathbf{N}_{0}\left[\mathrm{e}^{-F(s, u, w) 1_{\{s \leq \tau\}}}\left(1-\mathrm{e}^{-\lambda w(t-s)}\right)\right] \\
& \quad=\mathbf{N}_{0}\left[\mathrm{e}^{-F(s, u, w) 1_{\{s \leq \tau\}}}\left(1-\mathrm{e}^{-v_{t-r}(\lambda) w(r-s)}\right)\right]+h_{r-s} v_{t-r}(\lambda)-h_{t-s} \lambda .
\end{aligned}
$$

Then we can use (1.4) and continue the calculation

$$
\begin{aligned}
& \mathbf{P}\left[\exp \left\{-\int_{0}^{\tau} \int_{0}^{\infty} \int_{W} F(s, u, w) N_{0}(\mathrm{~d} s, \mathrm{~d} u, \mathrm{~d} w)-\lambda Z_{t}\right\}\right] \\
& =\exp \left\{-\lambda \int_{0}^{r} h_{t-s} \rho(s) \mathrm{d} s-\lambda \int_{r}^{t} h_{t-s} \rho(s) \mathrm{d} s\right\} \\
& \cdot \exp \left\{-\int_{0}^{r} \mathrm{~d} s \int_{0}^{\infty} \mathbf{N}_{0}\left(1-\mathrm{e}^{-F(s, u, w) 1_{\{s \leq \tau\}}}\right) \mathrm{d} u\right\} \\
& \cdot \exp \left\{-\int_{0}^{r} \mathrm{~d} s \int_{0}^{\rho(s)} \mathbf{N}_{0}\left[\mathrm{e}^{-F(s, u, w) 1_{\{s \leq \tau\}}}\left(1-\mathrm{e}^{-v_{t-r}(\lambda) w(r-s)}\right)\right] \mathrm{d} u\right\} \\
& \cdot \exp \left\{-\int_{0}^{r}\left[h_{r-s} v_{t-r}(\lambda)-h_{t-s} \lambda\right] \rho(s) \mathrm{d} s\right\} \\
& \cdot \exp \left\{-\int_{r}^{t} \rho(s) \mathrm{d} s \int_{(0, \infty)}\left(1-\mathrm{e}^{-y \lambda}\right) l_{t-s}(\mathrm{~d} y)\right\} \\
& =\exp \left\{-v_{t-r}(\lambda) \int_{0}^{r} h_{r-s} \rho(s) \mathrm{d} s-\int_{r}^{t} v_{t-s}(\lambda) \rho(s) \mathrm{d} s\right\} \\
& \cdot \exp \left\{-\int_{0}^{r} \mathrm{~d} s \int_{0}^{\infty} \mathbf{N}_{0}\left(1-\mathrm{e}^{-F(s, u, w) 1_{\{s \leq \tau\}}}\right) \mathrm{d} u\right\} \\
& \cdot \exp \left\{-\int_{0}^{r} \mathrm{~d} s \int_{0}^{\infty} \mathbf{N}_{0}\left[\mathrm{e}^{-F(s, u, w) 1_{\{s \leq \tau\}}}\left(1-\mathrm{e}^{-v_{t-r}(\lambda) w(r-s) 1_{\{u \leq \rho(s)\}}}\right)\right] \mathrm{d} u\right\} \\
& =\exp \left\{-v_{t-r}(\lambda) \int_{0}^{r} h_{r-s} \rho(s) \mathrm{d} s-\int_{r}^{t} v_{t-s}(\lambda) \rho(s) \mathrm{d} s\right\} \\
& \cdot \exp \left\{-\int_{0}^{r} \mathrm{~d} s \int_{0}^{\infty} \mathrm{N}_{0}\left(1-\mathrm{e}^{-F(s, u, w) 1_{\{s \leq \tau\}}-v_{t-r}(\lambda) w(r-s) 1_{\{u \leq \rho(s)\}}}\right) \mathrm{d} u\right\} \\
& =\exp \left\{-v_{t-r}(\lambda) \int_{0}^{r} h_{r-s} \rho(s) \mathrm{d} s-\int_{r}^{t} v_{t-s}(\lambda) \rho(s) \mathrm{d} s\right\} \\
& \cdot \mathbf{P}\left[\operatorname { e x p } \left\{-\int_{0}^{r} \int_{0}^{\infty} \int_{W}\left[F(s, u, w) 1_{\{s \leq \tau\}}\right.\right.\right. \\
& \left.\left.\left.+v_{t-r}(\lambda) w(r-s) 1_{\{u \leq \rho(s)\}}\right] N_{0}(\mathrm{~d} s, \mathrm{~d} u, \mathrm{~d} w)\right\}\right]
\end{aligned}
$$




$$
\begin{aligned}
&= \exp \left\{-v_{t-r}(\lambda) \int_{0}^{r} h_{r-s} \rho(s) \mathrm{d} s-\int_{r}^{t} v_{t-s}(\lambda) \rho(s) \mathrm{d} s\right\} \\
& \mathbf{P}\left[\operatorname { e x p } \left\{-\int_{0}^{\tau} \int_{0}^{\infty} \int_{W} F(s, u, w) N_{0}(\mathrm{~d} s, \mathrm{~d} u, \mathrm{~d} w)\right.\right. \\
&= \mathbf{P}\left[\exp \left\{-\int_{0}^{r} \int_{0}^{\rho(s)} \int_{W} v_{t-r}(\lambda) w(r-s) N_{0}(\mathrm{~d} s, \mathrm{~d} u, \mathrm{~d} w)\right\}\right] \\
& \int_{W}^{\infty} F(s, u, w) N_{0}(\mathrm{~d} s, \mathrm{~d} u, \mathrm{~d} w) \\
&\left.\left.-v_{t-r}(\lambda) Z_{r}-\int_{r}^{t} v_{t-s}(\lambda) \rho(s) \mathrm{d} s\right\}\right] .
\end{aligned}
$$

Then $\left\{\left(Z_{t}, \mathscr{H}_{t}^{0}\right)\right\}$ is a Markov process with transition semigroup $\left(P_{r, t}^{\rho}\right)_{t \geq r \geq 0}$. Using the independence of $\left\{\left(X_{t}, \mathscr{F}_{t}\right)\right\}$ and $\left\{\left(Z_{t}, \mathscr{H}_{t}^{0}\right)\right\}$, one can see $\left\{\left(Y_{t}, \mathscr{G}_{t}\right)\right\}$ is a Markov process with transition semigroup $\left(P_{r, t}^{\rho}\right)_{t \geq r \geq 0}$.

Theorem 3.2 Let $s \mapsto \rho(s)$ be a positive locally integrable function on $[0, \infty)$ and $(s, z) \mapsto$ $g(s, z)$ a positive measurable function on $[0, \infty) \times(0, \infty)$ such that

$$
\int_{0}^{t} \mathrm{~d} s \int_{(0, \infty)} g(s, z) z \nu(\mathrm{d} z)<\infty, \quad t \geq 0 .
$$

For $t \geq 0$ let

$$
\begin{aligned}
Y_{t}=X_{t} & +\int_{0}^{t} h_{t-s} \rho(s) \mathrm{d} s+\int_{0}^{t} \int_{0}^{\rho(s)} \int_{W} w(t-s) N_{0}(\mathrm{~d} s, \mathrm{~d} u, \mathrm{~d} w) \\
& +\int_{0}^{t} \int_{0}^{\infty} \int_{0}^{g(s, z)} \int_{W} w(t-s) N_{1}(\mathrm{~d} s, \mathrm{~d} z, \mathrm{~d} u, \mathrm{~d} w)
\end{aligned}
$$

and let $\mathscr{G}_{t}=\sigma\left(\mathscr{F}_{t} \cup \mathscr{H}_{t}^{0} \cup \mathscr{H}_{t}^{1}\right)$, where $\mathscr{H}_{t}^{0}$ is defined by (3.2) and

$$
\mathscr{H}_{t}^{1}=\sigma\left(\left\{N_{1}((0, s] \times C \times A): 0<s \leq t, C \in \mathscr{B}(0, \infty)^{2}, A \in \mathscr{W}_{t-s}\right\}\right) .
$$

Then $\left\{\left(Y_{t}, \mathscr{G}_{t}\right): t \geq 0\right\}$ is a Markov process in $[0, \infty)$ with inhomogeneous transition semigroup $\left(P_{r, t}^{\rho, g}\right)_{t \geq r \geq 0}$ given by

$$
\begin{aligned}
\int_{[0, \infty)} \mathrm{e}^{-\lambda y} P_{r, t}^{\rho, g}(x, \mathrm{~d} y)=\exp & \left\{-x v_{t-r}(\lambda)-\int_{r}^{t} v_{t-s}(\lambda) \rho(s) \mathrm{d} s\right. \\
& \left.-\int_{r}^{t} \mathrm{~d} s \int_{(0, \infty)}\left(1-\mathrm{e}^{-z v_{t-s}(\lambda)}\right) g(s, z) \nu(\mathrm{d} z)\right\} .
\end{aligned}
$$

Proof. Let $Z_{t}$ denote the last term on the right-hand side of (3.4). Let $t \geq r \geq \tau \geq 0$ and let $F$ be a positive function on $(0, \infty)^{3} \times W$ measurable relative to $\mathscr{B}[0, \tau] \times \mathscr{B}(0, \infty)^{2} \times \mathscr{W}_{r-\tau}$. For $\lambda \geq 0$, writing $H(s, z, u, w)=F(s, z, u, w) 1_{\{s \leq \tau\}}+\lambda w(t-s) 1_{\{u \leq g(s, z)\}}$, we have

$$
\mathbf{P}\left[\exp \left\{-\int_{0}^{\tau} \int_{0}^{\infty} \int_{0}^{\infty} \int_{W} F(s, z, u, w) N_{1}(\mathrm{~d} s, \mathrm{~d} z, \mathrm{~d} u, \mathrm{~d} w)-\lambda Z_{t}\right\}\right]
$$




$$
\begin{aligned}
& =\mathbf{P}\left[\exp \left\{-\int_{0}^{t} \int_{0}^{\infty} \int_{0}^{\infty} \int_{W} H(s, z, u, w) N_{1}(\mathrm{~d} s, \mathrm{~d} z, \mathrm{~d} u, \mathrm{~d} w)\right\}\right] \\
& =\exp \left\{-\int_{0}^{t} \mathrm{~d} s \int_{0}^{\infty} \nu(\mathrm{d} z) \int_{0}^{\infty} \mathbf{Q}_{z}\left(1-\mathrm{e}^{-H(s, z, u, w)}\right) \mathrm{d} u\right\} \\
& =\exp \left\{-\int_{0}^{t} \mathrm{~d} s \int_{0}^{\infty} \nu(\mathrm{d} z) \int_{0}^{\infty} \mathbf{Q}_{z}\left(1-\mathrm{e}^{-F(s, z, u, w) 1_{\{s \leq \tau\}}}\right) \mathrm{d} u\right. \\
& \left.-\int_{0}^{t} \mathrm{~d} s \int_{0}^{\infty} \nu(\mathrm{d} z) \int_{0}^{g(s, z)} \mathrm{Q}_{z}\left[\mathrm{e}^{-F(s, z, u, w) 1_{\{s \leq \tau\}}}\left(1-\mathrm{e}^{-\lambda w(t-s)}\right)\right] \mathrm{d} u\right\} \\
& =\exp \left\{-\int_{0}^{r} \mathrm{~d} s \int_{0}^{\infty} \nu(\mathrm{d} z) \int_{0}^{\infty} \mathbf{Q}_{z}\left(1-\mathrm{e}^{-F(s, z, u, w) 1_{\{s \leq \tau\}}}\right) \mathrm{d} u\right. \\
& -\int_{0}^{r} \mathrm{~d} s \int_{0}^{\infty} \nu(\mathrm{d} z) \int_{0}^{g(s, z)} \mathbf{Q}_{z}\left[\mathrm{e}^{-F(s, z, u, w) 1_{\{s \leq \tau\}}}\left(1-\mathrm{e}^{-v_{t-r}(\lambda) w(r-s)}\right)\right] \mathrm{d} u \\
& \left.-\int_{r}^{t} \mathrm{~d} s \int_{0}^{\infty} \nu(\mathrm{d} z) \int_{0}^{g(s, z)} \mathbf{Q}_{z}\left(1-\mathrm{e}^{-\lambda w(t-s)}\right) \mathrm{d} u\right\} \\
& =\mathbf{P}\left[\operatorname { e x p } \left\{-\int_{0}^{r} \int_{0}^{\infty} \int_{0}^{\infty} \int_{W}\left[F(s, z, u, w) 1_{\{s \leq \tau\}}\right.\right.\right. \\
& \left.+v_{t-r}(\lambda) w(t-s) 1_{\{u \leq g(s, z)\}}\right] N_{1}(\mathrm{~d} s, \mathrm{~d} z, \mathrm{~d} u, \mathrm{~d} w) \\
& \left.\left.-\int_{r}^{t} \mathrm{~d} s \int_{0}^{\infty}\left(1-\mathrm{e}^{-z v_{t-s}(\lambda)}\right) g(s, z) \nu(\mathrm{d} z)\right\}\right] \\
& =\mathbf{P}\left[\operatorname { e x p } \left\{-\int_{0}^{\tau} \int_{0}^{\infty} \int_{0}^{\infty} \int_{W} F(s, z, u, w) N_{1}(\mathrm{~d} s, \mathrm{~d} z, \mathrm{~d} u, \mathrm{~d} w)\right.\right. \\
& \left.\left.-v_{t-r}(\lambda) Z_{r}-\int_{r}^{t} \mathrm{~d} s \int_{0}^{\infty}\left(1-\mathrm{e}^{-z v_{t-s}(\lambda)}\right) g(s, z) \nu(\mathrm{d} z)\right\}\right] .
\end{aligned}
$$

Then $\left\{\left(Z_{t}, \mathscr{H}_{t}^{1}\right)\right\}$ is a Markov process in $[0, \infty)$ with inhomogeneous transition semigroup $\left(P_{r, t}^{0, g}\right)_{t \geq r \geq 0}$ defined by (3.5) with $\rho=0$. By Theorem 3.1 we see $\left\{\left(Y_{t}-Z_{t}, \sigma\left(\mathscr{F}_{t} \cup \mathscr{H}_{t}^{0}\right)\right)\right\}$ is a Markov process in $[0, \infty)$ with inhomogeneous transition semigroup $\left(P_{r, t}^{\rho, 0}\right)_{t \geq r \geq 0}$ defined by (3.5) with $g=0$. Then the desired result follows by the independence of those two processes.

We may think of the process $\left\{Y_{t}: t \geq 0\right\}$ defined by (3.4) as an inhomogeneous CBIprocess with immigration rates given by $\{(\rho(s), g(s, z)): s \geq 0, z>0\}$.

\section{Predictable immigration rates}

Suppose that $\left\{\left(X_{t}, \mathscr{F}_{t}\right)\right\},\left\{N_{0}(\mathrm{~d} s, \mathrm{~d} u, \mathrm{~d} w)\right\}$ and $\left\{N_{1}(\mathrm{~d} s, \mathrm{~d} z, \mathrm{~d} u, \mathrm{~d} w)\right\}$ are given as in the introduction. Let the filtration $\left(\mathscr{G}_{t}\right)$ be defined as in Theorem 3.2. Let $\mathscr{L}^{1}$ denote the set of $\left(\mathscr{G}_{t}\right)$-predictable processes $\rho=\{\rho(t): t \geq 0\}$ satisfying

$$
\|\rho\|_{t}:=\mathbf{P}\left[\int_{0}^{t}|\rho(s)| \mathrm{d} s\right]<\infty, \quad t \geq 0 .
$$


We identify $\rho_{1}, \rho_{2} \in \mathscr{L}^{1}$ if $\left\|\rho_{1}-\rho_{2}\right\|_{t}=0$ for every $t \geq 0$ and define the metric $d$ on $\mathscr{L}^{1}$ by

$$
d\left(\rho_{1}, \rho_{2}\right)=\sum_{n=1}^{\infty} \frac{1}{2^{n}}\left(1 \wedge\left\|\rho_{1}-\rho_{2}\right\|_{n}\right) .
$$

Let $\mathscr{L}_{\nu}^{1}(0, \infty)$ denote the set of two-parameter processes $g=\{g(t, z): t \geq 0, z>0\}$ that are $\left(\mathscr{G}_{t}\right)$-predictable in the sense of $\mathrm{Li}(2011$, p.163) and satisfy

$$
\|g\|_{\nu, t}:=\mathbf{P}\left[\int_{0}^{t} \int_{(0, \infty)}|g(s, z)| z \nu(\mathrm{d} z) \mathrm{d} s\right]<\infty, \quad t \geq 0 .
$$

We identify $g_{1}, g_{2} \in \mathscr{L}_{\nu}^{1}(0, \infty)$ if $\left\|g_{1}-g_{2}\right\|_{\nu, t}=0$ for every $t \geq 0$ and define the metric $d_{\nu}$ on $\mathscr{L}_{\nu}^{1}(0, \infty)$ by

$$
d_{\nu}\left(g_{1}, g_{2}\right)=\sum_{n=1}^{\infty} \frac{1}{2^{n}}\left(1 \wedge\left\|g_{1}-g_{2}\right\|_{\nu, n}\right)
$$

For $\rho \in \mathscr{L}^{1}, g \in \mathscr{L}_{\nu}^{1}(0, \infty)$ and a positive càdlàg process $\left\{Y_{t}: t \geq 0\right\}$ we consider the following properties:

(4.A) The process $\left\{Y_{t}: t \geq 0\right\}$ has no negative jumps and the optional random measure

$$
N_{0}(\mathrm{~d} s, \mathrm{~d} z):=\sum_{s>0} 1_{\left\{\Delta Y_{s} \neq 0\right\}} \delta_{\left(s, \Delta Y_{s}\right)}(\mathrm{d} s, \mathrm{~d} z),
$$

where $\Delta Y_{s}=Y_{s}-Y_{s-}$, has predictable compensator

$$
\hat{N}_{0}(\mathrm{~d} s, \mathrm{~d} z)=Y_{s-} \mathrm{d} s m(\mathrm{~d} z)+g(s, z) \mathrm{d} s \nu(\mathrm{d} z)=Y_{s} \mathrm{~d} s m(\mathrm{~d} z)+g(s, z) \mathrm{d} s \nu(\mathrm{d} z) .
$$

Let $\tilde{N}_{0}(\mathrm{~d} s, \mathrm{~d} z)=N_{0}(\mathrm{~d} s, \mathrm{~d} z)-\hat{N}_{0}(\mathrm{~d} s, \mathrm{~d} z)$. We have

$$
Y_{t}=Y_{0}+M^{c}(t)+M^{d}(t)+\int_{0}^{t}\left[\rho(s)+\int_{(0, \infty)} g(s, z) z \nu(\mathrm{d} z)-b Y_{s}\right] \mathrm{d} s,
$$

where $\left\{M^{c}(t): t \geq 0\right\}$ is a square-integrable continuous martingale with quadratic variation $2 c Y_{s-} \mathrm{d} s=2 c Y_{s} \mathrm{~d} s$ and

$$
M^{d}(t)=\int_{0}^{t} \int_{0}^{\infty} z \tilde{N}_{0}(\mathrm{~d} s, \mathrm{~d} z), \quad t \geq 0
$$

is a purely discontinuous martingale.

(4.B) For every $f \in \mathscr{D}$, we have

$$
\begin{aligned}
f\left(Y_{t}\right)= & f\left(Y_{0}\right)+\int_{0}^{t}\left[\rho(s) f^{\prime}\left(Y_{s}\right)-b Y_{s} f^{\prime}\left(Y_{s}\right)+c Y_{s} f^{\prime \prime}\left(Y_{s}\right)\right] \mathrm{d} s \\
& +\int_{0}^{t} \mathrm{~d} s \int_{(0, \infty)}^{t}\left[f\left(Y_{s}+z\right)-f\left(Y_{s}\right)\right] g(s, z) \nu(\mathrm{d} z)+\text { local mart. } \\
& +\int_{0}^{t} Y_{s} \mathrm{~d} s \int_{(0, \infty)}\left[f\left(Y_{s}+z\right)-f\left(Y_{s}\right)-z f^{\prime}\left(Y_{s}\right)\right] m(\mathrm{~d} z) .
\end{aligned}
$$


Proposition 4.1 The above properties (4.A) and (4.B) are equivalent.

Proof. If $\left\{Y_{t}\right\}$ has property (4.A), we may use Itô's formula to see it has property (4.B). Conversely, let us assume $\left\{Y_{t}\right\}$ has property (4.B). For any $\lambda>0$, by applying this property to the function $f(x)=\mathrm{e}^{-\lambda x}$ we have

$$
\begin{aligned}
\mathrm{e}^{-\lambda Y_{t}}= & \mathrm{e}^{-\lambda Y_{0}}+\int_{0}^{t} \mathrm{e}^{-\lambda Y_{s}}\left[b Y_{s} \lambda-\rho(s) \lambda+c Y_{s} \lambda^{2}\right] \mathrm{d} s \\
& +\int_{0}^{t} g(s, z) \mathrm{d} s \int_{(0, \infty)} \mathrm{e}^{-\lambda Y_{s}}\left(\mathrm{e}^{-\lambda z}-1\right) \nu(\mathrm{d} z)+\text { local mart. } \\
& +\int_{0}^{t} Y_{s} \mathrm{~d} s \int_{(0, \infty)} \mathrm{e}^{-\lambda Y_{s}}\left(\mathrm{e}^{-\lambda z}-1+\lambda z\right) m(\mathrm{~d} z) .
\end{aligned}
$$

Then the strictly positive process $\left\{\mathrm{e}^{-\lambda Y_{t}}: t \geq 0\right\}$ is a special semi-martingale. By Itô's formula, we see $\left\{Y_{t}: t \geq 0\right\}$ is also a special semi-martingale. Now define an optional random measure $N_{0}(\mathrm{~d} s, \mathrm{~d} z)$ on $(0, \infty) \times \mathbb{R}$ by

$$
N_{0}(\mathrm{~d} s, \mathrm{~d} z)=\sum_{s>0} 1_{\left\{\Delta Y_{s} \neq 0\right\}} \delta_{\left(s, \Delta Y_{s}\right)}(\mathrm{d} s, \mathrm{~d} z)
$$

where $\Delta Y_{s}=Y_{s}-Y_{s-}$. Let $\hat{N}_{0}(\mathrm{~d} s, \mathrm{~d} z)$ denote the predictable compensator of $N_{0}(\mathrm{~d} s, \mathrm{~d} z)$ and let $\tilde{N}_{0}(\mathrm{~d} s, \mathrm{~d} z)$ denote the compensated random measure; see Dellacherie and Meyer (1982, pp.375). We can write

$$
Y_{t}=Y_{0}+U_{t}+M_{t}^{c}+M_{t}^{d}
$$

where $\{U(t)\}$ is a predictable process with locally bounded variations, $\left\{M_{t}^{c}\right\}$ is a continuous local martingale and

$$
M^{d}(t)=\int_{0}^{t} \int_{\mathbb{R}} z \tilde{N}_{0}(\mathrm{~d} s, \mathrm{~d} z)
$$

is a purely discontinuous local martingale; see Dellacherie and Meyer (1982, p.353 and p.376) or Jacod and Shiryaev (2003, pp.84-85). Let $\left\{C_{t}\right\}$ denote the quadratic variation process of $\left\{M_{t}^{c}\right\}$. By (4.5) and Itô's formula,

$$
\begin{aligned}
\mathrm{e}^{-\lambda Y_{t}}= & \mathrm{e}^{-\lambda Y_{0}}-\lambda \int_{0}^{t} \mathrm{e}^{-\lambda Y_{s-}} \mathrm{d} U(s)+\frac{1}{2} \lambda^{2} \int_{0}^{t} \mathrm{e}^{-\lambda Y_{s-}} \mathrm{d} C_{s} \\
& +\int_{0}^{t} \int_{\mathbb{R}} \mathrm{e}^{-\lambda Y_{s-}}\left(\mathrm{e}^{-z \lambda}-1+z \lambda\right) N_{0}(\mathrm{~d} s, \mathrm{~d} z)+\text { local mart. } \\
= & \mathrm{e}^{-\lambda Y_{0}}-\lambda \int_{0}^{t} \mathrm{e}^{-\lambda Y_{s-}} \mathrm{d} U(s)+\frac{1}{2} \lambda^{2} \int_{0}^{t} \mathrm{e}^{-\lambda Y_{s-}} \mathrm{d} C_{s} \\
& +\int_{0}^{t} \int_{\mathbb{R}} \mathrm{e}^{-\lambda Y_{s-}}\left(\mathrm{e}^{-z \lambda}-1+z \lambda\right) \hat{N}_{0}(\mathrm{~d} s, \mathrm{~d} z)+\text { local mart. }
\end{aligned}
$$

But, the canonical decomposition of the special semi-martingale $\left\{\mathrm{e}^{-\lambda Y_{t}}: t \geq 0\right\}$ is unique; see, e.g., Dellacherie and Meyer (1982, p.213). From (4.4) and (4.6) we see $\mathrm{d} C_{s}=2 c Y_{s} \mathrm{~d} s$,

$$
\hat{N}_{0}(\mathrm{~d} s, \mathrm{~d} z)=Y_{s} \mathrm{~d} s m(\mathrm{~d} z)+g(s, z) \mathrm{d} s \nu(\mathrm{d} z)
$$


and

$$
\mathrm{d} U(s)=\left[\rho(s)-b Y_{s}+\int_{(0, \infty)} g(s, z) z \nu(\mathrm{d} z)\right] \mathrm{d} s .
$$

Then the process $\left\{Y_{t}\right\}$ has no negative jumps.

Now let us consider a kind of stochastic immigration rates. Given positive processes $\rho \in \mathscr{L}^{1}$ and $g \in \mathscr{L}_{\nu}^{1}(0, \infty)$, we define

$$
\begin{aligned}
Y_{t}=X_{t} & +\int_{0}^{t} h_{t-s} \rho(s) \mathrm{d} s+\int_{0}^{t} \int_{0}^{\rho(s)} \int_{W} w(t-s) N_{0}(\mathrm{~d} s, \mathrm{~d} u, \mathrm{~d} w) \\
& +\int_{0}^{t} \int_{0}^{\infty} \int_{0}^{g(s, z)} \int_{W} w(t-s) N_{1}(\mathrm{~d} s, \mathrm{~d} z, \mathrm{~d} u, \mathrm{~d} w) .
\end{aligned}
$$

Proposition 4.2 Let $\left\{Y_{t}: t \geq 0\right\}$ be defined by (4.7). Then for any $t \geq 0$ we have

$$
\mathbf{P}\left[Y_{t}\right]=\mathrm{e}^{-b t} \mathbf{P}\left[X_{0}\right]+\int_{0}^{t} \mathrm{e}^{-b(t-s)} \mathbf{P}\left[\rho(s)+\int_{0}^{\infty} g(s, z) z \nu(\mathrm{d} z)\right] \mathrm{d} s .
$$

Proof. Recall that both $s \mapsto \rho(s)$ and $(s, z) \mapsto g(s, z)$ are predictable. From (4.7) we have

$$
\begin{aligned}
Y_{t}=X_{t} & +\int_{0}^{t} h_{t-s} \rho(s) \mathrm{d} s+\int_{0}^{t} \int_{0}^{\rho(s)} \int_{W} w(t-s) \tilde{N}_{0}(\mathrm{~d} s, \mathrm{~d} u, \mathrm{~d} w) \\
& +\int_{0}^{t} \rho(s) \mathrm{d} s \int_{W} w(t-s) \mathbf{N}_{0}(\mathrm{~d} w) \\
& +\int_{0}^{t} \int_{0}^{\infty} \int_{0}^{g(s, z)} \int_{W} w(t-s) \tilde{N}_{1}(\mathrm{~d} s, \mathrm{~d} z, \mathrm{~d} u, \mathrm{~d} w) \\
& +\int_{0}^{t} \mathrm{~d} s \int_{0}^{\infty} g(s, z) \mathbf{Q}_{z}[w(t-s)] \nu(\mathrm{d} z) .
\end{aligned}
$$

By (3.7) in $\mathrm{Li} \mathrm{(2011)} \mathrm{or} \mathrm{(3.5)} \mathrm{in} \mathrm{Li}$ (2018b) we have $\mathbf{P}\left[X_{t}\right]=\mathbf{P}\left[X_{0}\right] \mathrm{e}^{-b t}=\mathbf{P}\left[Y_{0}\right] \mathrm{e}^{-b t}$. It follows that

$$
\begin{aligned}
\mathbf{P}\left[Y_{t}\right]= & \mathbf{P}\left[X_{t}\right]+\mathbf{P}\left[\int_{0}^{t} h_{t-s} \rho(s) \mathrm{d} s\right]+\mathbf{P}\left[\int_{0}^{t} \mathbf{N}_{0}[w(t-s)] \rho(s) \mathrm{d} s\right] \\
+ & \mathbf{P}\left[\int_{0}^{t} \mathrm{~d} s \int_{0}^{\infty} \mathbf{Q}_{z}[w(t-s)] g(s, z) \nu(\mathrm{d} z)\right] \\
= & \mathrm{e}^{-b t} \mathbf{P}\left[X_{0}\right]+\mathbf{P}\left[\int_{0}^{t}\left(h_{t-s}+\int_{0}^{\infty} y l_{t-s}(\mathrm{~d} y)\right) \rho(s) \mathrm{d} s\right] \\
+ & \mathbf{P}\left[\int_{0}^{t} \mathrm{e}^{-b(t-s)} \mathrm{d} s \int_{0}^{\infty} g(s, z) z \nu(\mathrm{d} z)\right] .
\end{aligned}
$$

Then we get desired equality from (3.4) in Li (2018b).

We may interpret the process $\left\{Y_{t}: t \geq 0\right\}$ defined by (4.7) as a generalization of the inhomogeneous CBI-process with predictable immigration rates given by $\{(\rho(s), g(s, z)): s \geq$ $0, z>0\}$. Recall that we identify $\rho_{1}, \rho_{2} \in \mathscr{L}^{1}$ if $\left\|\rho_{1}-\rho_{2}\right\|_{t}=0$ for every $t \geq 0$ and identify $g_{1}, g_{2} \in \mathscr{L}_{\nu}^{1}(0, \infty)$ if $\left\|g_{1}-g_{2}\right\|_{\nu, t}=0$ for every $t \geq 0$. By Proposition 4.2 one can see that choosing different representatives of $\rho \in \mathscr{L}^{1}$ and $g \in \mathscr{L}_{\nu}^{1}(0, \infty)$ in (4.7) only gives different modifications of the process $\left\{Y_{t}: t \geq 0\right\}$. 
Theorem 4.3 The process $\left\{Y_{t}: t \geq 0\right\}$ defined by (4.7) has a càdlàg modification and it satisfies properties (4.A) and (4.B).

The proof of the above theorem is based on approximations of $\rho \in \mathscr{L}^{1}$ and $g \in \mathscr{L}_{\nu}^{1}(0, \infty)$ using simpler processes. Let $\mathscr{L}^{0}$ denote the set of processes $\rho \in \mathscr{L}^{1}$ of the form

$$
\rho(s)=\rho\left(r_{1}\right) 1_{\{0\}}(s)+\sum_{i=0}^{\infty} \rho\left(r_{i+1}\right) 1_{\left(r_{i}, r_{i+1}\right]}(s),
$$

where $\left\{0=r_{0}<r_{1}<r_{2}<\cdots\right\}$ is a sequence increasing to infinity and each $\omega \mapsto \rho\left(\omega, r_{i+1}\right)$ is $\mathscr{G}_{r_{i}}$-measurable. Let $\mathscr{L}_{\nu}^{0}(0, \infty)$ denote the set of processes $g \in \mathscr{L}_{\nu}^{1}(0, \infty)$ of the form

$$
g(s, z)=g\left(r_{1}, z\right) 1_{\{0\}}(s)+\sum_{i=0}^{\infty} g\left(r_{i+1}, z\right) 1_{\left(r_{i}, r_{i+1}\right]}(s)
$$

where $\left\{0=r_{0}<r_{1}<r_{2}<\cdots\right\}$ is as above and each $(\omega, z) \mapsto g\left(\omega, r_{i+1}, z\right)$ is $\mathscr{G}_{r_{i}} \times \mathscr{B}(0, \infty)$ measurable.

Lemma 4.4 For positive processes $\rho \in \mathscr{L}^{0}$ and $g \in \mathscr{L}_{\nu}^{0}(0, \infty)$, the results of Theorem 4.3 hold.

Proof. By choosing $\left\{0=r_{0}<r_{1}<r_{2}<\cdots\right\}$ suitably, we can represent $s \mapsto \rho(s)$ and $(s, z) \mapsto g(s, z)$ by (4.9) and (4.10) using the same sequence. Then under $\mathbf{P}\left(\cdot \mid \mathscr{G}_{r_{i-1}}\right)$ we can think of $\rho\left(r_{i}\right)$ as a deterministic constant and $z \mapsto g\left(r_{i}, z\right)$ as a deterministic function. For $i \geq 1$ let

$$
\begin{aligned}
Y_{i}(t)=X_{t}+\int_{0}^{t \wedge r_{i}} & h_{t-s} \rho(s) \mathrm{d} s+\int_{0}^{t \wedge r_{i}} \int_{0}^{\rho(s)} \int_{W} w(t-s) N_{0}(\mathrm{~d} s, \mathrm{~d} u, \mathrm{~d} w) \\
& +\int_{0}^{t \wedge r_{i}} \int_{0}^{\infty} \int_{0}^{g(s, z)} \int_{W} w(t-s) N_{1}(\mathrm{~d} s, \mathrm{~d} z, \mathrm{~d} u, \mathrm{~d} w)
\end{aligned}
$$

and $\mathscr{G}_{t}^{i}=\sigma\left(\mathscr{F}_{t} \cup \mathscr{D}_{t}^{i}\right)$, where

$$
\begin{aligned}
& \mathscr{D}_{t}^{i}=\sigma\left(\left\{N _ { 0 } \left(\left[\left(0, s \wedge r_{i}\right] \times B \times A\right), N_{1}\left(\left(0, s \wedge r_{i}\right] \times C \times A\right):\right.\right.\right. \\
&\left.\left.0<s \leq t, B \in \mathscr{B}(0, \infty), C \in \mathscr{B}(0, \infty)^{2}, A \in \mathscr{W}_{t-s}\right\}\right) .
\end{aligned}
$$

Then $\left\{Y_{i}(t): t \geq 0\right\}$ is adapted to the filtration $\left\{\mathscr{G}_{t}^{i}: t \geq 0\right\}$. Note also that $Y_{i}(t)=Y_{t}, \mathscr{G}_{t}^{i}=\mathscr{G}_{t}$ for $0 \leq t \leq r_{i}$ and $Y_{i}(t) \leq Y_{t}, \mathscr{G}_{t}^{i} \subset \mathscr{G}_{t}$ for $t \geq r_{i}$. We claim that the following properties hold:

(a.1) $\left\{\left(Y_{i}(t), \mathscr{G}_{t}\right): r_{i-1} \leq t \leq r_{i}\right\}$ is a CBI-process under $\mathbf{P}\left(\cdot \mid \mathscr{G}_{r_{i-1}}\right)$ with time-independent immigration rate $\left\{\left(\rho\left(r_{i}\right), g\left(r_{i}, z\right)\right): z>0\right\}$;

(a.2) $\left\{\left(Y_{i}(t), \mathscr{G}_{t}\right): t \geq r_{i}\right\}$ and $\left\{\left(Y_{i}(t), \mathscr{G}_{t}^{i}\right): t \geq r_{i}\right\}$ are CB-processes under both $\mathbf{P}\left(\cdot \mid \mathscr{G}_{r_{i-1}}\right)$ and $\mathbf{P}\left(\cdot \mid \mathscr{G}_{r_{i}}\right)$. 
For $i=1$, those properties follow immediately from Theorem 3.2. Suppose they hold for some $i \geq 1$. Let

$$
\begin{gathered}
Z_{i}(t)=\int_{t \wedge r_{i}}^{t \wedge r_{i+1}} h_{t-s} \rho(s) \mathrm{d} s+\int_{t \wedge r_{i}}^{t \wedge r_{i+1}} \int_{0}^{\rho(s)} \int_{W} w(t-s) N_{0}(\mathrm{~d} s, \mathrm{~d} u, \mathrm{~d} w) \\
\quad+\int_{t \wedge r_{i}}^{t \wedge r_{i+1}} \int_{0}^{\infty} \int_{0}^{g(s, z)} \int_{W} w(t-s) N_{1}(\mathrm{~d} s, \mathrm{~d} z, \mathrm{~d} u, \mathrm{~d} w)
\end{gathered}
$$

and

$$
\begin{aligned}
\mathscr{H}_{t}^{i}=\sigma\left(\left\{N_{0}\left(\left(r_{i}, s \wedge r_{i+1}\right] \times B \times A\right), N_{1}\left(\left(r_{i}, s \wedge r_{i+1}\right] \times F \times A\right):\right.\right. & \\
& \left.\left.r_{i}<s \leq t, B \in \mathscr{B}(0, \infty), F \in \mathscr{B}(0, \infty)^{2}, A \in \mathscr{W}_{t-s}\right\}\right) .
\end{aligned}
$$

Using Theorem 2.3 again we see:

(b.1) $\left\{\left(Z_{i}(t), \mathscr{H}_{t}^{i}\right): r_{i} \leq t \leq r_{i+1}\right\}$ is a CBI-process under $\mathbf{P}\left(\cdot \mid \mathscr{G}_{r_{i}}\right)$ with time-independent immigration rate $\left.\left\{\rho\left(r_{i+1}\right), g\left(r_{i+1}, z\right)\right): z>0\right\}$;

(b.2) $\left\{\left(Z_{i}(t), \mathscr{H}_{t}^{i}\right): t \geq r_{i+1}\right\}$ is a CB-process under both $\mathbf{P}\left(\cdot \mid \mathscr{G}_{r_{i}}\right)$ and $\mathbf{P}\left(\cdot \mid \mathscr{G}_{r_{i+1}}\right)$.

Here the processes $\left\{\left(Y_{i}(t), \mathscr{G}_{t}^{i}\right): t \geq r_{i}\right\}$ and $\left\{\left(Z_{i}(t), \mathscr{H}_{t}^{i}\right): t \geq r_{i}\right\}$ are independent of each other under $\mathbf{P}\left(\cdot \mid \mathscr{G}_{r_{i}}\right)$. Note also that $Y_{i+1}(t)=Y_{i}(t)+Z_{i}(t)$ and $\mathscr{G}_{t}^{i+1}=\sigma\left(\mathscr{G}_{t}^{i} \cup \mathscr{H}_{t}^{i}\right)$ for $t \geq r_{i}$. By Proposition 5.7 in Li (2018b), properties (a.1) and (a.2) also hold when $i$ is replaced by $i+1$. Then they hold for all $i \geq 1$ by induction. By applying Theorem 7.2 in Li (2018b) step by step on the intervals $\left[r_{i-1}, r_{i}\right], i=1,2, \cdots$ we see $\left\{Y_{t}: t \geq 0\right\}$ satisfies property (4.B). By Proposition 4.1 it also satisfies property (4.A).

Lemma 4.5 Suppose that $\rho \in \mathscr{L}^{1}$ and $g \in \mathscr{L}_{\nu}^{1}(0, \infty)$ are positive processes. Let $\left\{\rho_{k}\right\} \subset \mathscr{L}^{0}$ and $\left\{g_{k}\right\} \subset \mathscr{L}_{\nu}^{0}(0, \infty)$ be positive sequences such that $d\left(\rho_{k}, \rho\right)+d_{\nu}\left(g_{k}, g\right) \rightarrow 0$ as $k \rightarrow \infty$. Let $\left\{Y_{k}(t): t \geq 0\right\}$ be the positive càdlàg process defined by (4.7) with $\rho=\rho_{k}$ and $g=g_{k}$. Then there is a positive càdlàg process $\{Y(t): t \geq 0\}$ so that

$$
\lim _{k \rightarrow \infty} \mathbf{P}\left[\sup _{0 \leq s \leq t}\left|Y_{k}(s)-Y(s)\right|\right]=0, \quad t \geq 0,
$$

and there is a subsequence $\left\{k_{n}\right\} \subset\{k\}$ so that a.s.

$$
\lim _{n \rightarrow \infty} \sup _{0 \leq s \leq t}\left|Y_{k_{n}}(s)-Y(s)\right|=0, \quad t \geq 0 .
$$

Proof. For any $j, k \geq 1$, we can represent $\rho_{j}, \rho_{k}$ and $g_{j}, g_{k}$ in the form of (4.9) and (4.10) using the same sequence $\left\{0=r_{0}<r_{1}<r_{2}<\cdots\right\}$. Then we have $\left|Y_{j}(t)-Y_{k}(t)\right| \leq Z_{j, k}(t)$, where

$$
\begin{gathered}
Z_{j, k}(t)=\int_{0}^{t} h_{t-s}\left|\rho_{j}(s)-\rho_{k}(s)\right| \mathrm{d} s+\int_{0}^{t} \int_{\rho_{j}(s) \wedge \rho_{k}(s)}^{\rho_{j}(s) \vee \rho_{k}(s)} \int_{W} w(t-s) N_{0}(\mathrm{~d} s, \mathrm{~d} u, \mathrm{~d} w) \\
\quad+\int_{0}^{t} \int_{0}^{\infty} \int_{g_{j}(s, z) \wedge g_{k}(s, z)}^{g_{j}(s, z) \vee g_{k}(s, z)} \int_{W} w(t-s) N_{1}(\mathrm{~d} s, \mathrm{~d} z, \mathrm{~d} u, \mathrm{~d} w)
\end{gathered}
$$


We can rewrite the above expression into

$$
\begin{gathered}
Z_{j, k}(t)=\int_{0}^{t} h_{t-s}\left|\rho_{j}(s)-\rho_{k}(s)\right| \mathrm{d} s+\int_{0}^{t} \int_{0}^{\left|\rho_{j}(s)-\rho_{k}(s)\right|} \int_{W} w(t-s) N_{0}^{j, k}(\mathrm{~d} s, \mathrm{~d} u, \mathrm{~d} w) \\
+\int_{0}^{t} \int_{0}^{\infty} \int_{0}^{\left|g_{j}(s, z)-g_{k}(s, z)\right|} \int_{W} w(t-s) N_{1}^{j, k}(\mathrm{~d} s, \mathrm{~d} z, \mathrm{~d} u, \mathrm{~d} w)
\end{gathered}
$$

where $N_{0}^{j, k}(\mathrm{~d} s, \mathrm{~d} u, \mathrm{~d} w)=N_{0}\left(\mathrm{~d} s, \rho_{j}(s) \wedge \rho_{k}(s)+\mathrm{d} u, \mathrm{~d} w\right)$ is a Poisson random measure with intensity $\mathrm{d} s \mathrm{~d} u \mathbf{N}_{0}(\mathrm{~d} w)$ and $N_{1}^{j, k}(\mathrm{~d} s, \mathrm{~d} z, \mathrm{~d} u, \mathrm{~d} w)=N_{1}\left(\mathrm{~d} s, \mathrm{~d} z, g_{j}(s, z) \wedge g_{k}(s, z)+\mathrm{d} u, \mathrm{~d} w\right)$ is a Poisson random measure with intensity $\mathrm{d} s \nu(\mathrm{d} z) \mathrm{d} u \mathbf{Q}_{z}(\mathrm{~d} w)$. One can see that $\left\{\left(Z_{j, k}(t), \mathscr{G}_{t}\right)\right.$ : $t \geq 0\}$ is a CBI-process with predictable immigration rates given by $\left\{\left(\left|\rho_{j}(s)-\rho_{k}(s)\right|, \mid g_{j}(s, z)-\right.\right.$ $\left.\left.g_{k}(s, z) \mid\right): s \geq 0, z>0\right\}$. By Proposition 4.2 we see that

$$
\mathbf{P}\left[Z_{j, k}(t)\right] \leq \mathrm{e}^{|b| t}\left(\left\|\rho_{j}-\rho_{k}\right\|_{t}+\left\|g_{j}-g_{k}\right\|_{\nu, t}\right) .
$$

By Lemma 4.4, the results of Theorem 4.3 hold for $\left\{\left(Z_{j, k}(t), \mathscr{G}_{t}\right)\right\}$. By Proposition 4.1, this process has properties (4.A) and (4.B). In particular, it has no negative jumps and the optional random measure

$$
N_{j, k}(\mathrm{~d} s, \mathrm{~d} z)=\sum_{s>0} 1_{\left\{\Delta Z_{j, k}(s) \neq 0\right\}} \delta_{\left(s, \Delta Z_{j, k}(s)\right)}(\mathrm{d} s, \mathrm{~d} z),
$$

where $\Delta Z_{j, k}(s)=Z_{j, k}(s)-Z_{j, k}(s-)$, has predictable compensator

$$
\hat{N}_{j, k}(\mathrm{~d} s, \mathrm{~d} z)=Z_{j, k}(s) \mathrm{d} s m(\mathrm{~d} z)+\left|g_{k}(s, z)-g_{j}(s, z)\right| \mathrm{d} s \nu(\mathrm{d} z) .
$$

Let $\tilde{N}_{j, k}(\mathrm{~d} s, \mathrm{~d} z)=N_{j, k}(\mathrm{~d} s, \mathrm{~d} z)-\hat{N}_{j, k}(\mathrm{~d} s, \mathrm{~d} z)$ be the compensated random measure. We have

$$
\begin{gathered}
Z_{j, k}(t)=M_{j, k}^{c}(t)+M_{j, k}^{d}(t)+\int_{0}^{t}\left[\left|\rho_{j}(s)-\rho_{k}(s)\right|-b Z_{j, k}(s)\right] \mathrm{d} s \\
+\int_{0}^{t} \mathrm{~d} s \int_{0}^{\infty}\left|g_{j}(s, z)-g_{k}(s, z)\right| z \nu(\mathrm{d} z),
\end{gathered}
$$

where $\left\{M_{j, k}^{c}(t): t \geq 0\right\}$ is a continuous local martingale with quadratic variation $2 c Z_{j, k}(t) \mathrm{d} t$ and

$$
M_{j, k}^{d}(t)=\int_{0}^{t} \int_{0}^{\infty} z \tilde{N}_{j, k}(\mathrm{~d} s, \mathrm{~d} z)
$$

is a purely discontinuous local martingale. Using Hölder's inequality and Doob's martingale inequality we get

$$
\begin{aligned}
\mathbf{P}\left[\sup _{0 \leq s \leq t} Z_{j, k}(s)\right] \leq & \mathbf{P}\left[\int_{0}^{t}\left|\rho_{j}(s)-\rho_{k}(s)\right| \mathrm{d} s\right]+|b| \mathbf{P}\left[\int_{0}^{t} Z_{j, k}(s) \mathrm{d} s\right] \\
& +\mathbf{P}\left[\int_{0}^{t} \mathrm{~d} s \int_{0}^{\infty}\left|g_{j}(s, z)-g_{k}(s, z)\right| z \nu(\mathrm{d} z) \mathrm{d} s\right] \\
& +\mathbf{P}\left[\sup _{0 \leq s \leq t}\left|M_{j, k}^{c}(t)\right|\right]+\mathbf{P}\left[\sup _{0 \leq s \leq t}\left|\int_{0}^{s} \int_{0}^{\infty} z \tilde{N}(\mathrm{~d} r, \mathrm{~d} z)\right|\right]
\end{aligned}
$$




$$
\begin{aligned}
\leq & \left\|\rho_{j}-\rho_{k}\right\|_{t}+\left\|g_{j}-g_{k}\right\|_{\nu, t}+|b| \mathbf{P}\left[\int_{0}^{t} Z_{j, k}(s) \mathrm{d} s\right] \\
& +\mathbf{P}\left[\sup _{0 \leq s \leq t}\left|M_{j, k}^{c}(t)\right|\right]+\mathbf{P}\left[\sup _{0 \leq s \leq t}\left|\int_{0}^{s} \int_{0}^{1} z \tilde{N}(\mathrm{~d} r, \mathrm{~d} z)\right|\right] \\
& +\mathbf{P}\left[\left|\int_{0}^{t} \int_{1}^{\infty} z N(\mathrm{~d} r, \mathrm{~d} z)\right|+\left|\int_{0}^{t} \int_{1}^{\infty} z \hat{N}(\mathrm{~d} r, \mathrm{~d} z)\right|\right] \\
\leq & \left\|\rho_{j}-\rho_{k}\right\|_{t}+\left\|g_{j}-g_{k}\right\|_{\nu, t}+|b| \mathbf{P}\left[\int_{0}^{t} Z_{j, k}(s) \mathrm{d} s\right] \\
& +2\left\{c \mathbf{P}\left[\int_{0}^{t} Z_{j, k}(s) \mathrm{d} s\right]\right\}^{\frac{1}{2}}+2\left\{\mathbf{P}\left[\int_{0}^{t} Z_{j, k}(s) \mathrm{d} s \int_{0}^{1} u^{2} m(\mathrm{~d} u)\right]\right\}^{\frac{1}{2}} \\
& +2\left\{\mathbf{P}\left[\int_{0}^{t} \mathrm{~d} s \int_{0}^{1}\left|g_{j}(s, z)-g_{k}(s, z)\right| z^{2} \nu(\mathrm{d} z)\right]\right\}^{\frac{1}{2}} \\
& +2 \mathbf{P}\left[\int_{0}^{t} Z_{j, k}(s) \mathrm{d} s \int_{1}^{\infty} u m(\mathrm{~d} u)\right] \\
& +2 \mathbf{P}\left[\int_{0}^{t} \mathrm{~d} s \int_{1}^{\infty}\left|g_{j}(s, z)-g_{k}(s, z)\right| z \nu(\mathrm{d} z)\right] .
\end{aligned}
$$

By (4.14) one can find a locally bounded function $t \mapsto C(t)$ so that

$$
\mathbf{P}\left[\sup _{0 \leq s \leq t} Z_{j, k}(s)\right] \leq C(t)\left(\left\|\rho_{j}-\rho_{k}\right\|_{t}+\left\|g_{j}-g_{k}\right\|_{\nu, t}+\sqrt{\left\|\rho_{j}-\rho_{k}\right\|_{t}}+\sqrt{\left\|g_{j}-g_{k}\right\|_{\nu, t}}\right) .
$$

It follows that

$$
\lim _{j, k \rightarrow \infty} \mathbf{P}\left[\sup _{0 \leq s \leq t}\left|Y_{j}(s)-Y_{k}(s)\right|\right] \leq \lim _{j, k \rightarrow \infty} \mathbf{P}\left[\sup _{0 \leq s \leq t} Z_{j, k}(s)\right]=0 .
$$

Then there is an increasing sequence of integers $\left\{k_{n}\right\}$ such that

$$
\mathbf{P}\left[\sup _{0 \leq s \leq n}\left|Y_{j}(s)-Y_{k}(s)\right|\right] \leq 1 / 2^{n}, \quad j, k \geq k_{n}
$$

Consequently, for every $t \geq 0$,

$$
\mathbf{P}\left[\sum_{n=1}^{\infty} \sup _{0 \leq s \leq t}\left|Y_{k_{n}}(s)-Y_{k_{n+1}}(s)\right|\right]<\infty .
$$

Thus we have a.s.

$$
\sum_{n=1}^{\infty} \sup _{0 \leq s \leq t}\left|Y_{k_{n}}(s)-Y_{k_{n+1}}(s)\right|<\infty .
$$

That yields the existence of a càdlàg positive process $\{Y(t): t \geq 0\}$ so that (4.12) holds a.s. for every $t \geq 0$. By letting $j \rightarrow \infty$ along the sequence $\left\{k_{n}\right\}$ in (4.16) we get

$$
\mathbf{P}\left[\sup _{0 \leq s \leq n}\left|Y(s)-Y_{k}(s)\right|\right] \leq 1 / 2^{n}, \quad k \geq k_{n} .
$$


Then we get 4.11).

Proof of Theorem 4.3 By Proposition 10.3 in $\mathrm{Li}$ (2011, p.236), there are positive sequences $\left\{\rho_{k}\right\} \subset \mathscr{L}^{0}$ and $\left\{g_{k}\right\} \subset \mathscr{L}_{\nu}^{0}(0, \infty)$ such that $d\left(\rho_{k}, \rho\right)+d_{\nu}\left(g_{k}, g\right) \rightarrow 0$ as $k \rightarrow \infty$. Let $\left\{Y_{k}(t): t \geq 0\right\}$ be defined by (4.7) with $\rho=\rho_{k}$ and $g=g_{k}$. By Lemma 4.5, there is a positive càdlàg process $\{Y(t): t \geq 0\}$ and a sequence $\left\{k_{n}\right\}$ so that (4.11) and (4.12) hold for every $t \geq 0$. For each $k \geq 1$ we have $\left|Y_{t}-Y_{k}(t)\right| \leq Z_{k}(t)$, where

$$
\begin{gathered}
Z_{k}(t)=\int_{0}^{t} h_{t-s}\left|\rho(s)-\rho_{k}(s)\right| \mathrm{d} s+\int_{0}^{t} \int_{\rho(s) \wedge \rho_{k}(s)}^{\rho(s) \vee \rho_{k}(s)} \int_{W} w(t-s) N_{0}(\mathrm{~d} s, \mathrm{~d} u, \mathrm{~d} w) \\
\quad+\int_{0}^{t} \int_{0}^{\infty} \int_{g(s, z) \wedge g_{k}(s, z)}^{g(s, z) \vee g_{k}(s, z)} \int_{W} w(t-s) N_{1}(\mathrm{~d} s, \mathrm{~d} z, \mathrm{~d} u, \mathrm{~d} w) .
\end{gathered}
$$

By the arguments leading to (4.14) we get

$$
\mathbf{P}\left[\left|Y_{t}-Y_{k}(t)\right|\right] \leq \mathbf{P}\left[Z_{k}(t)\right] \leq \mathrm{e}^{|b| t}\left(\left\|\rho-\rho_{k}\right\|_{t}+\left\|g-g_{k}\right\|_{\nu, t}\right) .
$$

It follows that $\mathbf{P}\left[\left|Y_{t}-Y_{k}(t)\right|\right] \rightarrow 0$ as $n \rightarrow \infty$. By choosing a smaller sequence $\left\{k_{n}\right\}$ we have a.s. $Y_{t}=\lim _{n \rightarrow \infty} Y_{k_{n}}(t)=Y(t)$. Then the positive càdlàg process $\{Y(t): t \geq 0\}$ is a modification of $\left\{Y_{t}: t \geq 0\right\}$. By Lemma 4.4 property (4.B) holds for $\left\{Y_{k}(t): t \geq 0\right\}$ with $\rho=\rho_{k}$ and $g=g_{k}$. Then the property holds for $\{Y(t): t \geq 0\}$. By Proposition 4.1 the process $\{Y(t): t \geq 0\}$ also has property (4.A).

Proposition 4.6 Suppose that $\rho_{k}, \rho \in \mathscr{L}^{1}$ and $g_{k}, g \in \mathscr{L}_{\nu}^{1}(0, \infty)$ are positive processes such that $d\left(\rho_{k}, \rho\right)+d_{\nu}\left(g_{k}, g\right) \rightarrow 0$ as $k \rightarrow \infty$. Let $\left\{Y_{t}: t \geq 0\right\}$ be the positive càdlàg process defined by (4.7). Let $\left\{Y_{k}(t): t \geq 0\right\}$ be the positive càdlàg process defined by the same formula with $\rho=\rho_{k}$. Then we have

$$
\lim _{k \rightarrow \infty} \mathbf{P}\left[\sup _{0 \leq s \leq t}\left|Y_{k}(s)-Y_{s}\right|\right]=0, \quad t \geq 0,
$$

and there is a subsequence $\left\{k_{n}\right\} \subset\{k\}$ so that a.s.

$$
\lim _{n \rightarrow \infty} \sup _{0 \leq s \leq t}\left|Y_{k_{n}}(s)-Y_{s}\right|=0, \quad t \geq 0 .
$$

Proof. For $j, k \geq 1$ let $\left\{Z_{j, k}(t): t \geq 0\right\}$ be defined as in (4.13). Then $\left\{\left(Z_{j, k}(t), \mathscr{G}_{t}\right): t \geq 0\right\}$ is a CBI-process with predictable immigration rates given by $\left\{\left(\left|\rho_{j}(s)-\rho_{k}(s)\right|,\left|g_{j}(s, z)-g_{k}(s, z)\right|\right)\right.$ : $s \geq 0, z>0\}$. By Proposition 4.1 and Theorem 4.3, the properties (4.A) and (4.B) hold for $\left\{Z_{j, k}(t): t \geq 0\right\}$. In particular, a decomposition like 4.15) is valid for this process. The remaining arguments go as in the proof of Lemma 4.5 .

\section{Solutions of the stochastic equations}

In this section, we prove there is a pathwise unique solution to (1.13) and show the solution is a Markov process with generator $(L, \mathscr{D})$. Suppose that $\left\{\left(X_{t}, \mathscr{F}_{t}\right)\right\},\left\{N_{0}(\mathrm{~d} s, \mathrm{~d} u, \mathrm{~d} w)\right\}$ and $\left\{N_{1}(\mathrm{~d} s, \mathrm{~d} z, \mathrm{~d} u, \mathrm{~d} w)\right\}$ are given as in the introduction. Let the filtration $\left(\mathscr{G}_{t}\right)$ be defined as in Theorem 3.2. The following result gives a reformulation of (1.10) in terms of a martingale problem. 
Proposition 5.1 A positive càdlàg process $\left\{Y_{t}: t \geq 0\right\}$ is a weak solution of (1.10) if and only if it solves the martingale problem of $(L, \mathscr{D})$, that is, for every $f \in \mathscr{D}$,

$$
f\left(Y_{t}\right)=f\left(Y_{0}\right)+\int_{0}^{t} L f\left(Y_{s}\right) \mathrm{d} s+\text { local mart }
$$

Proof. If $\left\{Y_{t}: t \geq 0\right\}$ is a weak solution of (1.10), we may use Itô's formula to see it solves the martingale problem given by (5.1). Conversely, let us assume $\left\{Y_{t}: t \geq 0\right\}$ is a solution of the martingale problem (5.1). By Proposition 4.1, the process has property (4.A) in Section 4 with $\rho(s)=\beta\left(Y_{s-}\right)$ and $g(s, z)=q\left(Y_{s-}, z\right)$. By Theorem III.7.1' in Ikeda and Watanabe (1989, p.90), on an extension of the original probability space there is a Brownian motion $\{B(s)\}$ so that

$$
M^{c}(t)=\int_{0}^{t} \sqrt{2 c Y_{s-}} \mathrm{d} B(s), \quad t \geq 0
$$

By Theorem III.7.4 in Ikeda and Watanabe (1989, p.93), on a further extension of the probability space there are independent Poisson time-space random measures $M(\mathrm{~d} s, \mathrm{~d} z, \mathrm{~d} u)$ and $N(\mathrm{~d} s, \mathrm{~d} z)$ with intensities $\mathrm{d} s m(\mathrm{~d} z) \mathrm{d} u$ and $\mathrm{d} s \nu(\mathrm{d} z)$, respectively, so that

$\int_{0}^{t} \int_{0}^{\infty} z \tilde{N}_{0}(\mathrm{~d} s, \mathrm{~d} z)=\int_{0}^{t} \int_{0}^{\infty} \int_{0}^{Y_{s-}} z \tilde{M}(\mathrm{~d} s, \mathrm{~d} z, \mathrm{~d} u)+\int_{0}^{t} \int_{0}^{\infty} \int_{0}^{g\left(Y_{s-}, z\right)} z N(\mathrm{~d} s, \mathrm{~d} z, \mathrm{~d} u)$.

Then $\left\{Y_{t}\right\}$ is a weak solution of the stochastic equation 1.10).

Proposition 5.2 Let $\left\{Y_{t}: t \geq 0\right\}$ be a solution to (1.13). Then there is a locally bounded function $t \mapsto C(t)$ so that

$$
\mathbf{P}\left[Y_{t}\right] \leq C(t)\left(1+\mathbf{P}\left[X_{0}\right]+\sqrt{\mathbf{P}\left[X_{0}\right]}\right), \quad t \geq 0 .
$$

Proof. Let $\left\{\tau_{k}: k \geq 1\right\}$ be the increasing sequence of stopping times defined by $\tau_{k}=\inf \{t \geq$ $\left.0: Y_{t} \geq k\right\}$. Then we have a.s. $\lim _{k \rightarrow \infty} \tau_{k}=\infty$. By (1.13) and Condition (1.A) we have

$$
\begin{aligned}
\mathbf{P}\left[Y_{t} 1_{\left\{t<\tau_{k}\right\}}\right] \leq & \mathbf{P}\left[X_{t \wedge \tau_{k}}\right]+\mathbf{P}\left[\int_{0}^{t \wedge \tau_{k}}\left(h_{t-s}+\mathbf{N}_{0}[w(t-s)]\right) \beta\left(Y_{s-}\right) \mathrm{d} s\right] \\
& +\mathbf{P}\left[\int_{0}^{t \wedge \tau_{k}} \mathrm{~d} s \int_{0}^{\infty} \mathbf{Q}_{z}[w(t-s)] q\left(Y_{s-}, z\right) \nu(\mathrm{d} z)\right] \\
\leq & \mathbf{P}\left[X_{t \wedge \tau_{k}}\right]+\mathbf{P}\left[\int_{0}^{t \wedge \tau_{k}} \mathrm{e}^{-b(t-s)}\left(\beta\left(Y_{s-}\right)+\int_{0}^{\infty} q\left(Y_{s-}, z\right) z \nu(\mathrm{d} z)\right) \mathrm{d} s\right] \\
\leq & \mathbf{P}\left[\sup _{0 \leq s \leq t} X_{s}\right]+K \mathbf{P}\left[\int_{0}^{t} \mathrm{e}^{-b(t-s)}\left(1+Y_{s-}\right) 1_{\left\{s<\tau_{k}\right\}} \mathrm{d} s\right] .
\end{aligned}
$$

By Corollary 7.3 in $\mathrm{Li}(2018 \mathrm{~b})$, there is a locally bounded function $t \mapsto C_{0}(t)$ so that

$$
\mathbf{P}\left[\sup _{0 \leq s \leq t} X_{s}\right] \leq C_{0}(t)\left(\mathbf{P}\left[X_{0}\right]+\sqrt{\mathbf{P}\left[X_{0}\right]}\right) .
$$


Then $t \mapsto \mathbf{P}\left[Y_{t} 1_{\left\{t<\tau_{k}\right\}}\right]$ is locally bounded and

$$
\begin{aligned}
\mathbf{P}\left[Y_{t} 1_{\left\{t<\tau_{k}\right\}}\right] & =C_{0}(t)\left(\mathbf{P}\left[X_{0}\right]+\sqrt{\mathbf{P}\left[X_{0}\right]}\right)+K \mathbf{P}\left[\int_{0}^{t} \mathrm{e}^{-b(t-s)}\left(1+Y_{s}\right) 1_{\left\{s<\tau_{k}\right\}} \mathrm{d} s\right] \\
& \leq C_{0}(t)\left(\mathbf{P}\left[X_{0}\right]+\sqrt{\mathbf{P}\left[X_{0}\right]}\right)+K t \mathrm{e}^{|b| t}+K \mathrm{e}^{|b| t} \int_{0}^{t} \mathbf{P}\left[Y_{s} 1_{\left\{s<\tau_{k}\right\}}\right] \mathrm{d} s .
\end{aligned}
$$

By Gronwall's inequality, we can can find a function $t \mapsto C(t)$ independent of $k \geq 1$ so that

$$
\mathbf{P}\left[Y_{t} 1_{\left\{t<\tau_{k}\right\}}\right] \leq C(t)\left(1+\mathbf{P}\left[X_{0}\right]+\sqrt{\mathbf{P}\left[X_{0}\right]}\right) .
$$

Then (5.2) follows from (5.3) by Fatou's lemma.

Proposition 5.3 There is at most one solution to (1.13).

Proof. Suppose that $\left\{Y_{t}: t \geq 0\right\}$ and $\left\{Z_{t}: t \geq 0\right\}$ are two solutions of the equation. Then we have $\left|Y_{t}-Z_{t}\right| \leq \xi_{t} \leq Y_{t}+Z_{t}$, where

$$
\begin{aligned}
\xi_{t}=\int_{0}^{t} h_{t-s}\left|\beta\left(Y_{s-}\right)-\beta\left(Z_{s-}\right)\right| \mathrm{d} s+\int_{0}^{t} \int_{\beta\left(Y_{s-}\right) \wedge \beta\left(Z_{s-}\right)}^{\beta\left(Y_{s-}\right) \vee \beta\left(Z_{s-}\right)} \int_{W} w(t-s) N_{0}(\mathrm{~d} s, \mathrm{~d} u, \mathrm{~d} w) \\
+\int_{0}^{t} \int_{0}^{\infty} \int_{q\left(Y_{s-}, z\right) \wedge q\left(Z_{s-}, z\right)}^{q\left(Y_{s-}, z\right) \vee q\left(Z_{s-}, z\right)} \int_{W} w(t-s) N_{1}(\mathrm{~d} s, \mathrm{~d} z, \mathrm{~d} u, \mathrm{~d} w)
\end{aligned}
$$

By Proposition 5.2, the function $t \mapsto \mathbf{P}\left[Y_{t}+Z_{t}\right]=\mathbf{P}\left[Y_{t}\right]+\mathbf{P}\left[Z_{t}\right]$ is locally bounded, then so is $t \mapsto \mathbf{P}\left[\xi_{t}\right]$. We can rewrite (5.4) into

$$
\begin{gathered}
\xi_{t}=\int_{0}^{t} h_{t-s}\left|\beta\left(Y_{s-}\right)-\beta\left(Z_{s-}\right)\right| \mathrm{d} s+\int_{0}^{t} \int_{0}^{\left|\beta\left(Y_{s-}\right)-\beta\left(Z_{s-}\right)\right|} \int_{W} w(t-s) M_{0}(\mathrm{~d} s, \mathrm{~d} u, \mathrm{~d} w) \\
+\int_{0}^{t} \int_{0}^{\infty} \int_{0}^{\left|q\left(Y_{s-}, z\right)-q\left(Z_{s-}, z\right)\right|} \int_{W} w(t-s) M_{1}(\mathrm{~d} s, \mathrm{~d} z, \mathrm{~d} u, \mathrm{~d} w),
\end{gathered}
$$

where $M_{0}(\mathrm{~d} s, \mathrm{~d} u, \mathrm{~d} w)$ is a spatial shift of $N_{0}(\mathrm{~d} s, \mathrm{~d} u, \mathrm{~d} w)$ and $M_{0}(\mathrm{~d} s, \mathrm{~d} u, \mathrm{~d} w)$ is a spatial shift of $N_{1}(\mathrm{~d} s, \mathrm{~d} z, \mathrm{~d} u, \mathrm{~d} w)$. By Proposition 4.2 and Condition (1.B),

$$
\begin{aligned}
\mathbf{P}\left[\xi_{t}\right] & =\mathbf{P}\left[\int_{0}^{t} \mathrm{e}^{-b(t-s)}\left(\left|\beta\left(Y_{s-}\right)-\beta\left(Z_{s-}\right)\right|+\int_{0}^{\infty}\left|q\left(Y_{s-}, z\right)-q\left(Z_{s-}, z\right)\right| z \nu(\mathrm{d} z)\right) \mathrm{d} s\right] \\
& \leq \mathrm{e}^{|b| t} \mathbf{P}\left[\int_{0}^{t} r\left(\left|Y_{s-}-Z_{s-}\right|\right) \mathrm{d} s\right] \leq \mathrm{e}^{|b| t} \int_{0}^{t} r\left(\mathbf{P}\left[\left|Y_{s}-Z_{s}\right|\right]\right) \mathrm{d} s \\
& \leq \mathrm{e}^{|b| t} \int_{0}^{t} r\left(\mathbf{P}\left[\xi_{s}\right]\right) \mathrm{d} s,
\end{aligned}
$$

where the last two inequalities hold by the convexity and monotonicity of $u \mapsto r(u)$. Now define the continuous increasing function

$$
u(t)=\int_{0}^{t} r\left(\mathbf{P}\left[\xi_{s}\right]\right) \mathrm{d} s, \quad t \geq 0 .
$$


We claim that $u(t)=0$ for every $t \geq 0$. Otherwise, there is $a>0$ so that $u(a)>0$. Let $v=\inf \{t \in[0, a]: u(t)>0\}$. Then $0 \leq v<a$ and $u(v)=0$. Since $u \mapsto r(u)$ is increasing, we get $\mathrm{d} u(s)=r\left(\mathbf{P}\left[\xi_{s}\right]\right) \mathrm{d} s \leq r\left(\mathrm{e}^{|b| s} u(s)\right) \mathrm{d} s$ by (5.5). For $v<t \leq a$ we have

$$
a-t \geq \int_{t}^{a} \frac{r\left(\mathrm{e}^{|b| s} u(s)\right) \mathrm{d} s}{r\left(\mathrm{e}^{|b| a} u(s)\right)} \geq \int_{t}^{a} \frac{\mathrm{d} u(s)}{r\left(\mathrm{e}^{|b| a} u(s)\right)}=\mathrm{e}^{-|b| a} \int_{\mathrm{e}^{|b| a} u(t)}^{\mathrm{e}^{|b| a} u(a)} \frac{\mathrm{d} u}{r(u)} .
$$

By letting $t \rightarrow v$ and using Condition (1.B) we conclude

$$
a-v \geq \mathrm{e}^{-|b| a} \int_{0}^{\mathrm{e}^{|b| a} u(a)} r(u)^{-1} \mathrm{~d} u=\infty
$$

which gives a contradiction. Then we must have $u(t)=0$ and hence $\mathbf{P}\left[\xi_{t}\right]=0$ for every $t \geq 0$. That proves the pathwise uniqueness of solution to (1.13).

Theorem 5.4 There is a pathwise unique solution $\left\{Y_{t}: t \geq 0\right\}$ to (1.13). Moreover, the process $\left\{\left(Y_{t}, \mathscr{G}_{t}\right): t \geq 0\right\}$ solves the martingale problem (5.1) for $(L, \mathscr{D})$.

Proof. The pathwise uniqueness of solution to (1.13) follows from Proposition 5.3, We shall give the construction of a solution to the stochastic equation in two steps.

Step 1. Instead of (1.A), we assume the following stronger condition: there is a constant $K \geq 0$ so that

$$
\beta(x)+\int_{(0, \infty)} q(x, z) z \nu(\mathrm{d} z) \leq K, \quad x \geq 0 .
$$

Let $Y_{0}(t)=X_{t}$ and define inductively

$$
\rho_{k}(s)=\beta\left(Y_{k-1}(s-)\right), g_{k}(s, z)=q\left(Y_{k-1}(s-), z\right)
$$

and

$$
\begin{aligned}
Y_{k}(t)=X_{t} & +\int_{0}^{t} h_{t-s} \rho_{k}(s) \mathrm{d} s+\int_{0}^{t} \int_{0}^{\rho_{k}(s)} \int_{W} w(t-s) N_{0}(\mathrm{~d} s, \mathrm{~d} u, \mathrm{~d} w) \\
& +\int_{0}^{t} \int_{0}^{\infty} \int_{0}^{g_{k}(s, z)} \int_{W} w(t-s) N_{1}(\mathrm{~d} s, \mathrm{~d} z, \mathrm{~d} u, \mathrm{~d} w) .
\end{aligned}
$$

Then by Proposition4.2 we one can show

$$
\mathbf{P}\left[Y_{k}(t)\right] \leq C_{1}(t):=\mathrm{e}^{-b t} \mathbf{P}\left[X_{0}\right]+K \int_{0}^{t} \mathrm{e}^{-b(t-s)} \mathrm{d} s .
$$

For $k, j \geq 1$ let the process $\left\{Z_{j, k}(t): t \geq 0\right\}$ be defined as in (4.13). Then $\left|Y_{j}(t)-Y_{k}(t)\right| \leq$ $Z_{j, k}(t) \leq Y_{j}(t)+Y_{k}(t)$. From (5.7) it follows that $\mathbf{P}\left[Z_{j, k}(t)\right] \leq 2 C_{1}(t)$ for $t \geq 0$. As in (5.5) one can see

$$
\mathbf{P}\left[Z_{j, k}(t)\right] \leq \mathrm{e}^{|b| t} \int_{0}^{t} r\left(\mathbf{P}\left[\left|Y_{j-1}(s)-Y_{k-1}(s)\right|\right]\right) \mathrm{d} s \leq \mathrm{e}^{|b| t} \int_{0}^{t} r\left(\mathbf{P}\left[Z_{j-1, k-1}(s)\right]\right) \mathrm{d} s .
$$


Let $R_{n}(t)=\sup _{j, k \geq n} \mathbf{P}\left[Z_{j, k}(t)\right] \leq 2 C_{1}(t)$. By (5.8) and dominated convergence,

$$
\lim _{n \rightarrow \infty} R_{n}(t) \leq \mathrm{e}^{|b| t} \int_{0}^{t} r\left(\lim _{n \rightarrow \infty} R_{n-1}(s)\right) \mathrm{d} s=\mathrm{e}^{|b| t} \int_{0}^{t} r\left(\lim _{n \rightarrow \infty} R_{n}(s)\right) \mathrm{d} s .
$$

As in the last part of the proof of Proposition 5.3 we see

$$
\lim _{j, k \rightarrow \infty} \mathbf{P}\left[Z_{j, k}(t)\right]=\lim _{n \rightarrow \infty} R_{n}(t)=0 .
$$

Observe that

$$
\begin{aligned}
\left\|\rho_{j}-\rho_{k}\right\|_{t}+\left\|g_{j}-g_{k}\right\|_{t, \nu}= & \mathbf{P}\left[\int _ { 0 } ^ { t } \left(\left|\beta\left(Y_{j-1}(s-)\right)-\beta\left(Y_{k-1}(s-)\right)\right|\right.\right. \\
& \left.\left.+\int_{0}^{\infty}\left|q\left(Y_{j-1}(s-), z\right)-q\left(Y_{k-1}(s-), z\right)\right| z \nu(\mathrm{d} z)\right) \mathrm{d} s\right] \\
\leq & \mathrm{e}^{|b| t} \mathbf{P}\left[\int_{0}^{t} r\left(\left|Y_{j-1}(s)-Y_{k-1}(s)\right|\right) \mathrm{d} s\right] \\
\leq & \mathrm{e}^{|b| t} \int_{0}^{t} r\left(\mathbf{P}\left[Z_{j-1, k-1}(s)\right]\right) \mathrm{d} s .
\end{aligned}
$$

Then (5.9) implies that $\left\|\rho_{j}-\rho_{k}\right\|_{t}+\left\|g_{j}-g_{k}\right\|_{t, \nu} \rightarrow 0$ as $j, k \rightarrow \infty$. Since $\mathscr{L}^{1}$ and $\mathscr{L}_{\nu}^{1}(0, \infty)$ are complete, there are positive processes $\rho \in \mathscr{L}^{1}$ and $g \in \mathscr{L}_{\nu}^{1}(0, \infty)$ so that $\left\|\rho_{k}-\rho\right\|_{t}+\| g_{k}-$ $g \|_{t, \nu} \rightarrow 0$ as $k \rightarrow \infty$. Define the process $\left\{Y_{t}: t \geq 0\right\}$ by (4.7). By Condition (1.B) we have

$$
\begin{aligned}
\int_{0}^{t} \mathbf{P} & {\left[\left|\rho_{k}(s)-\beta\left(Y_{s-}\right)\right|+\int_{(0, \infty)}\left|g_{k}(s, z)-q\left(Y_{s-}, z\right)\right| z \nu(\mathrm{d} z)\right] \mathrm{d} s } \\
& =\int_{0}^{t} \mathbf{P}\left[\left|\beta\left(Y_{k-1}(s)\right)-\beta\left(Y_{s}\right)\right|+\int_{(0, \infty)}\left|q\left(Y_{k-1}(s), z\right)-q\left(Y_{s}, z\right)\right| z \nu(\mathrm{d} z)\right] \mathrm{d} s \\
& \leq \int_{0}^{t} \mathbf{P}\left[r\left(\left|Y_{k-1}(s)-Y_{s}\right|\right)\right] \mathrm{d} s \leq \int_{0}^{t} r\left(\mathbf{P}\left[\left|Y_{k-1}(s)-Y_{s}\right|\right]\right) \mathrm{d} s .
\end{aligned}
$$

By Proposition 4.6, the right-hand side vanishes as $k \rightarrow \infty$. Then we may identify $s \mapsto \rho(s)$ and $s \mapsto \beta(Y(s-))$ as elements of $\mathscr{L}^{1}$ and identify $(s, z) \mapsto g(s, z)$ and $(s, z) \mapsto q(Y(s-), z)$ as elements of $\mathscr{L}_{\nu}^{1}(0, \infty)$. Now (1.13) follows from (4.7). The martingale problem characterization (5.1) follows by Theorem 4.3.

Step 2. In the general case where (5.6) is not necessarily true, for $n \geq 1$ we consider the stochastic equation

$$
\begin{aligned}
Y_{t}=X_{t} & +\int_{0}^{t} h_{t-s} \beta\left(Y_{s-}\right) \mathrm{d} s+\int_{0}^{t} \int_{0}^{\beta\left(Y_{s-} \wedge n\right)} \int_{W} w(t-s) N_{0}(\mathrm{~d} s, \mathrm{~d} u, \mathrm{~d} w) \\
& +\int_{0}^{t} \int_{0}^{\infty} \int_{0}^{q\left(Y_{s-\wedge n, z)}\right.} \int_{W} w(t-s) N_{1}(\mathrm{~d} s, \mathrm{~d} z, \mathrm{~d} u, \mathrm{~d} w)
\end{aligned}
$$

By Step 1 and Proposition 5.3, there is a pathwise unique solution $\left\{Y_{n}(t): t \geq 0\right\}$ to (5.10). Let $\tau_{n}=\inf \left\{t \geq 0: Y_{n}(t) \geq n\right\}$. Then $Y_{n}(t)=Y_{n+1}(t)$ for $0 \leq t<\tau_{n}$. As in the proof of Proposition 5.2, one can see $\tau_{n} \rightarrow \infty$ increasingly in probability as $t \rightarrow \infty$. Then 
$Y(t)=\lim _{n \rightarrow \infty} Y_{k}(t)$ defines a process $\{Y(t): t \geq 0\}$. From (5.10) we see this process is a solution to (1.13).

Comparison properties of stochastic equations of the form (1.10) were studied in Bertoin and Le Gall (2006), Dawson and Li (2012) and Fu and Li (2010). Those results have played important roles in the study of stochastic flows induced by those equations. The next theorem provides a comparison result for the stochastic equation (1.13).

Theorem 5.5 Let $(\beta, q)$ and $\left(\beta^{\prime}, q^{\prime}\right)$ be two sets of parameters satisfying Conditions (1.A) and (1.B). Suppose that:

- $x \mapsto \beta(x)$ or $x \mapsto \beta^{\prime}(x)$ is increasing on $[0, \infty)$;

- for each $z>0, x \mapsto q(x, z)$ or $x \mapsto q^{\prime}(x, z)$ is increasing on $[0, \infty)$.

- $\beta(x) \leq \beta^{\prime}(x)$ and $q(x, z) \leq q^{\prime}(x, z)$ for all $x \geq 0$ and $z>0$.

Let $\left\{Y_{t}: t \geq 0\right\}$ be the solution of (1.13) and $\left\{Y_{t}^{\prime}: t \geq 0\right\}$ the solution of the stochastic equation with $(\beta, q)$ replaced by $\left(\beta^{\prime}, q^{\prime}\right)$. Then $\mathbf{P}\left(Y_{t} \leq Y_{t}^{\prime}\right.$ for all $\left.t \geq 0\right)=1$.

Proof. For simplicity, we assume $x \mapsto \beta(x)$ is increasing. Let $A=\{z>0: x \mapsto q(x, z)$ is increasing $\}$. Then $x \mapsto q^{\prime}(x, z)$ is increasing for $z \in A^{\prime}:=(0, \infty) \backslash A$. Let $\left\{Y_{k}(t)\right\}$ be the sequence defined as in the proof of Theorem 5.4 and let $\left\{Y_{k}^{\prime}(t)\right\}$ be defined in the same way with $(\beta, q)$ replaced by $\left(\beta^{\prime}, q^{\prime}\right)$. By induction in $k \geq 1$ we see that

$$
\begin{aligned}
Y_{k}(t)=X_{t}+\int_{0}^{t} h_{t-s} \beta\left(Y_{k-1}(s-)\right) \mathrm{d} s+\int_{0}^{t} \int_{0}^{\beta\left(Y_{k-1}(s-)\right)} \int_{W} w(t-s) N_{0}(\mathrm{~d} s, \mathrm{~d} u, \mathrm{~d} w) \\
\quad+\int_{0}^{t} \int_{A} \int_{0}^{q\left(Y_{k-1}(s-), z\right)} w(t-s) N_{1}(\mathrm{~d} s, \mathrm{~d} z, \mathrm{~d} u, \mathrm{~d} w) \\
\quad+\int_{0}^{t} \int_{A^{\prime}} \int_{0}^{q^{\prime}\left(Y_{k-1}(s-), z\right)} \int_{W} w(t-s) N_{1}(\mathrm{~d} s, \mathrm{~d} z, \mathrm{~d} u, \mathrm{~d} w) \\
\leq X_{t}+\int_{0}^{t} h_{t-s} \beta\left(Y_{k-1}^{\prime}(s-)\right) \mathrm{d} s+\int_{0}^{t} \int_{0}^{\beta\left(Y_{k-1}^{\prime}(s-)\right)} \int_{W} w(t-s) N_{0}(\mathrm{~d} s, \mathrm{~d} u, \mathrm{~d} w) \\
\quad+\int_{0}^{t} \int_{A} \int_{0}^{q\left(Y_{k-1}^{\prime}(s-), z\right)} \int_{W}^{q^{\prime}\left(Y_{k-1}^{\prime}(s-), z\right)} w(t-s) N_{1}(\mathrm{~d} s, \mathrm{~d} z, \mathrm{~d} u, \mathrm{~d} w) \\
\quad+\int_{0}^{t} \int_{A^{\prime}} \int_{0}^{t} w(t-s) N_{1}(\mathrm{~d} s, \mathrm{~d} z, \mathrm{~d} u, \mathrm{~d} w) \\
\leq X_{t}+\int_{0}^{t} h_{t-s} \beta^{\prime}\left(Y_{k-1}^{\prime}(s-)\right) \mathrm{d} s+\int_{0}^{\beta^{\prime}\left(Y_{k-1}^{\prime}(s-)\right)} \int_{0} w(t-s) N_{0}(\mathrm{~d} s, \mathrm{~d} u, \mathrm{~d} w) \\
+\int_{0}^{t} \int_{0}^{\infty} \int_{0}^{q^{\prime}\left(Y_{k-1}^{\prime}(s-), z\right)} \int_{W} w(t-s) N_{1}(\mathrm{~d} s, \mathrm{~d} z, \mathrm{~d} u, \mathrm{~d} w) \\
=Y_{k}^{\prime}(t) .
\end{aligned}
$$

Then $\mathbf{P}\left(Y_{t} \leq Y_{t}^{\prime}\right.$ for all $\left.t \geq 0\right)=1$ by the proof of Theorem 5.4 .

Acknowledgments I would like to thank Rongjuan Fang and an anonymous referee for their careful reading of the paper and very helpful comments on the presentation of the results. 
I am grateful to the Laboratory of Mathematics and Complex Systems (Ministry of Education) for providing the research facilities to carry out the project.

\section{References}

Aliev, S.A. (1985): A limit theorem for the Galton-Watson branching processes with immigration. Ukrainian Math. J. 37, 535-438.

Aliev, S.A. and Shchurenkov, V.M. (1982): Transitional phenomena and the convergence of GaltonWatson processes to Jiřina processes. Theory Probab. Appl. 27, 472-485.

Berestycki, J., Fittipaldi, M.C. and Fontbona, J. (2018): Ray-Knight representation of flows of branching processes with competition by pruning of Lévy trees. Probab. Theory Related Fields 172, 725-788.

Bernis, G. and Scotti, S. (2018+): Clustering effects through Hawkes processes. In: From Probability to Finance - Lecture note of BICMR Summer School on Financial Mathematics. Series of Mathematical Lectures from Peking University. Springer.

Bertoin, J. and Le Gall, J.-F. (2006): Stochastic flows associated to coalescent processes III: Limit theorems. Illinois J. Math. 50, 147-181.

Cox, J., Ingersoll, J. and Ross, S. (1985): A theory of the term structure of interest rate. Econometrica 53, 385-408.

Dawson, D.A. and Li, Z. (2003): Construction of immigration superprocesses with dependent spatial motion from one-dimensional excursions. Probab. Theory Related Fields 127, 37-61.

Dawson, D.A. and Li, Z. (2006): Skew convolution semigroups and affine markov processes. Ann. Probab. 34, 1103-1142.

Dawson, D.A. and Li, Z. (2012): Stochastic equations, flows and measure-valued processes. Ann. Probab. 40, 813-857.

Dellacherie, C. and Meyer, P.A. (1982): Probabilities and Potential. Chapters V-VIII. North-Holland, Amsterdam.

Feller, W. (1951): Diffusion processes in genetics. In: Proceedings 2nd Berkeley Symp. Math. Statist. Probab., 1950, 227-246. Univ. of California Press, Berkeley and Los Angeles.

Fu, Z. and Li, Z. (2004): Measure-valued diffusions and stochastic equations with Poisson process. Osaka J. Math. 41, 724-744.

$\mathrm{Fu}, \mathrm{Z}$. and Li, Z. (2010): Stochastic equations of non-negative processes with jumps. Stochastic Process. Appl. 120, 306-330.

Getoor, R.K. and Glover, J. (1987): Constructing Markov processes with random times of birth and death. In: Seminar on Stochastic Processes, 1986 (Charlottesville, Va., 1986), 35-69. Progr. Probab. Statist. 13. Birkhäuser, Boston, MA.

Grimvall, A. (1974): On the convergence of sequences of branching processes. Ann. Probab. 2, 10271045. 
Ikeda, N. and Watanabe, S. (1989): Stochastic Differential Equations and Diffusion Processes. 2nd Ed. North-Holland, Amsterdam; Kodansha, Tokyo.

Jacod, J. and Shiryaev, A.N. (2003): Limit Theorems for Stochastic Processes. 2nd Ed. Springer, Heidelberg.

Jiao, Y., Ma, C. and Scotti, S. (2017): Alpha-CIR model with branching processes in sovereign interest rate modeling. Finance Stochastics 21, 789-813.

Kawazu, K. and Watanabe, S. (1971): Branching processes with immigration and related limit theorems. Theory Probab. Appl. 16, 36-54.

Kuznetsov, S.E. (1974): Construction of Markov processes with random times of birth and death. Theory Probab. Appl. 18, 571-575.

Kyprianou, A.E. (2014): Fluctuations of Lévy Processes with Applications. 2nd Ed. Springer, Heidelberg.

Lambert, A. (2005): The branching process with logistic growth. Ann. Appl. Probab. 15, 1506-1535.

Lamperti, J. (1967a): The limit of a sequence of branching processes. Z. Wahrsch. verw. Geb. 7, 271-288.

Lamperti, J. (1967b): Continuous state branching processes. Bull. Amer. Math. Soc. 73, 382-386.

Li, P.S. (2018a): A continuous-state polynomial branching process. Stochastic Process. Appl. To appear.

Li, P.S., Yang, X. and Zhou, X. (2017+): A general continuous-state nonlinear branching process. arXiv:1708.01560.

Li, Z. (2006): A limit theorem for discrete Galton-Watson branching processes with immigration. $J$. Appl. Probab. 43, 289-295.

Li, Z. (2011): Measure-Valued Branching Markov Processes. Probability and its Applications. Springer, Heidelberg.

Li, Z. (2018b): Continuous-State Branching Processes with Immigration. In: From Probability to Finance - Lecture note of BICMR Summer School on Financial Mathematics, Series of Mathematical Lectures from Peking University. Springer. Available at: http://arxiv.org/abs/1901.03521v1.

Li, Z. and Ma, C. (2015): Asymptotic properties of estimators in a stable Cox-Ingersoll-Ross model. Stochastic Process. Appl. 125, 3196-3233.

Li, Z. and Zhang, W. (2019): Continuous-state branching processes with dependent immigration. Sci. China Math., Chinses Ed. To appear (In Chinese).

Pardoux, E. (2016): Probabilistic Models of Population Evolution: Scaling Limits, Genealogies and Interactions. Springer, Switzerland.

Situ, R. (2005): Theory of Stochastic Differential Equations with Jumps and Applications. Springer, Heidelberg. 